In cooperation with the Texas Water Development Board

\title{
Base Flow (1966-2005) and Streamflow Gain and Loss (2006) of the Brazos River, McLennan County to Fort Bend County, Texas, 2006
}
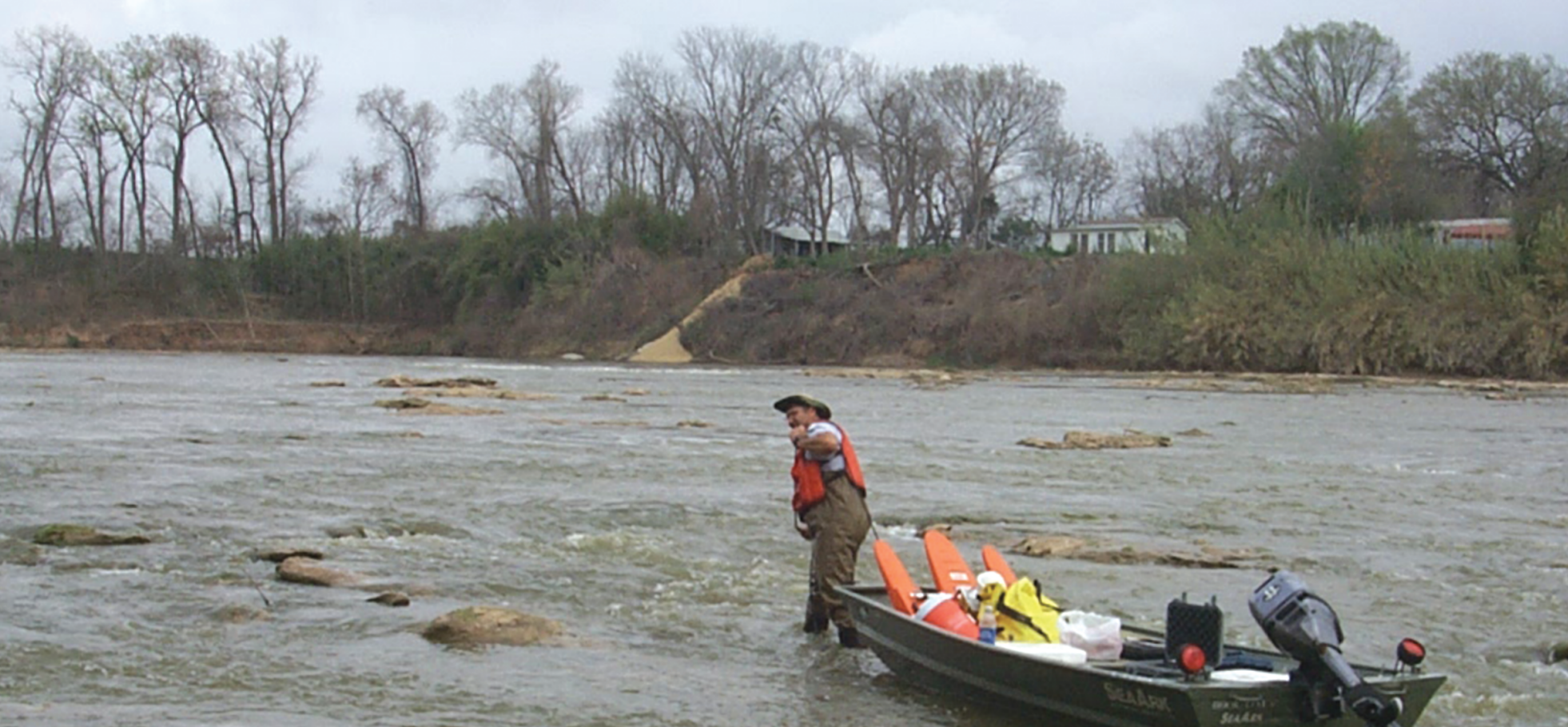

Scientific Investigations Report 2007-5286 
Cover: U.S. Geological Survey field technician deploying discharge measurement equipment at Hidalgo Falls on the Brazos River near Navasota, Texas, March 8, 2006 (photograph by Jimmy Hopkins, U.S. Geological Survey). 


\section{Base Flow (1966-2005) and Streamflow Gain and Loss (2006) of the Brazos River, McLennan County to Fort Bend County, Texas}

By Michael J. Turco, Jeffery W. East, and Matthew S. Milburn

In cooperation with the Texas Water Development Board

Scientific Investigations Report 2007-5286 


\section{U.S. Department of the Interior DIRK KEMPTHORNE, Secretary}

\section{U.S. Geological Survey \\ Mark D. Myers, Director}

\section{U.S. Geological Survey, Reston, Virginia: 2007}

For product and ordering information:

World Wide Web: http://www.usgs.gov/pubprod

Telephone: 1-888-ASK-USGS

For more information on the USGS — the Federal source for science about the Earth, its natural and living resources, natural hazards, and the environment:

World Wide Web: http://www.usgs.gov

Telephone: 1-888-ASK-USGS

Any use of trade, product, or firm names is for descriptive purposes only and does not imply endorsement by the U.S. Government.

Although this report is in the public domain, permission must be secured from the individual copyright owners to reproduce any copyrighted materials contained within this report.

Suggested citation:

Turco, M.J., East, J.W., and Milburn, M.S., 2007, Base flow (1966-2005) and streamflow gain and loss (2006) of the Brazos River, McLennan County to Fort Bend County, Texas: U.S. Geological Survey Scientific Investigations Report 2007-5286, $27 \mathrm{p}$. 


\section{Contents}

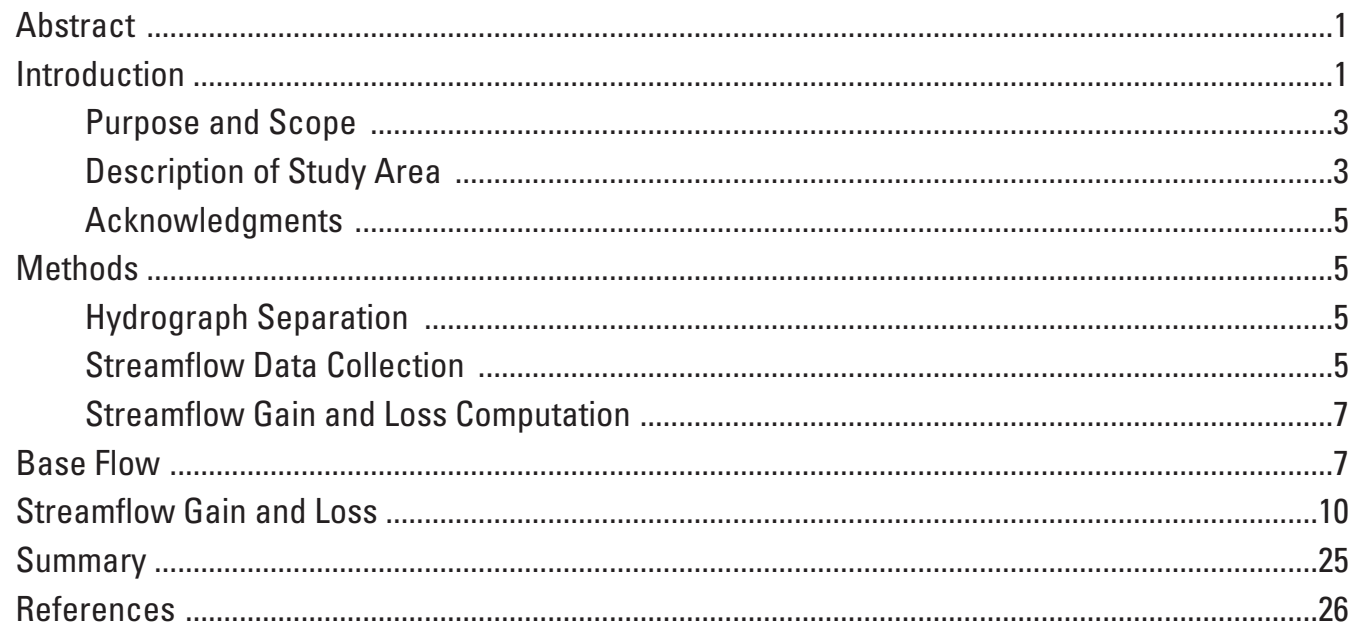

\section{Figures}

\section{1-2. Maps showing:}

1. Study area, Brazos River, and extent of Brazos River alluvium aquifer, McLennan County to Fort Bend County, Texas

2. Brazos River alluvium aquifer, McLennan County to Fort Bend County, Texas; outcrops of underlying aquifers; and data-collection sites

3-9. Graphs showing:

3. Base flow and runoff components of streamflow, 1966-2005, from hydrograph separation for (A) 08098290 Brazos River near Highbank, Texas; (B) 08111500 Brazos River near Hempstead, Texas; and (C) 08114000 Brazos River at Richmond, Texas

4. (A) Relation between annual base-flow index for 08098290 Brazos River near Highbank, Texas, and for 08111500 Brazos River near Hempstead, Texas; (B) relation between annual base-flow index for 08098290 Brazos River near Highbank, Texas, plus six inflow tributary sites, and for 08111500 Brazos River near Hempstead, Texas; (C) time series of annual base-flow index for 08111500 Brazos River near Hempstead, Texas, 1966-2005; and (D) time series of annual base-flow index for 08098290 Brazos River near Highbank, Texas, 1966-2005, and for 08098290 Brazos River near Highbank, Texas, plus six inflow tributary sites, 1966-2005

5. (A) Relation between annual base-flow index for 08111500 Brazos River near Hempstead, Texas, and for 08114000 Brazos River at Richmond, Texas; (B) relation between annual base-flow index for 08111500 Brazos River near Hempstead, Texas, plus one inflow tributary site, and for 08114000 Brazos River at Richmond, Texas; (C) time series of annual base-flow index for 08114000 Brazos River at Richmond, Texas, 1966-2005; and (D) time series of annual base-flow index for 08111500 Brazos River near Hempstead, Texas, 1966-2005, and for 08111500 Brazos River near Hempstead, Texas, plus one inflow tributary site, 1966-2005 
6. Daily mean streamflow for March 1-15, 2006, at (A) 08096500 Brazos River at Waco, Texas; (B) 08108700 Brazos River at State Highway 21 near Bryan, Texas; and (C) 08111500 Brazos River near Hempstead, Texas

7. Daily mean streamflow for August 5-20, 2006, at (A) 08096500 Brazos River at Waco, Texas; (B) 08108700 Brazos River at State Highway 21 near Bryan, Texas; and (C) 08111500 Brazos River near Hempstead, Texas 15

8. Instantaneous streamflow at 36 sites on the Brazos River, McLennan County to Fort Bend County, Texas, March 6-10, 2006 .16

9. Instantaneous streamflow at 28 sites on the Brazos River, McLennan County to Fort Bend County, Texas, August 10-18, 2006 16

10-11. Maps showing:

10. Reaches of the Brazos River, McLennan County to Fort Bend County, Texas, identified as gaining or losing streamflow, March 6-10, 2006

11. Reaches of the Brazos River, McLennan County to Fort Bend County, Texas, identified as gaining or losing streamflow, August 10-18, 2006.

\section{Tables}

1. U.S. Geological Survey streamflow-gaging stations on the Brazos River and selected tributaries, McLennan County to Fort Bend County, Texas, that provided data for hydrograph separation, water years 1966-2005 (October 1965 through September 2005)

2. Sites on the Brazos River and selected tributaries, McLennan County to Fort Bend County, Texas, at which measurements for streamflow gain and loss computation were made, March and August 2006

3. Results of hydrograph separation for 08098290 Brazos River near Highbank, Texas, 08111500 Brazos River near Hempstead, Texas, and 08114000 Brazos River at Richmond, Texas, 1966-2005

4. Results of hydrograph separation for 08098290 Brazos River near Highbank, Texas, plus six inflow tributary sites, 1966-2005; and 08111500 Brazos River near Hempstead, Texas, plus one inflow tributary site, 1966-2005

5. Instantaneous streamflow, water temperature, and specific conductance for sites on the Brazos River and selected tributaries, McLennan County to Fort Bend County, Texas, March 6-10, 2006

6. Instantaneous streamflow for sites on the Brazos River and selected tributaries, McLennan County to Fort Bend County, Texas, August 10-18, 2006

7. Streamflow gains and losses computed for 35 reaches on the Brazos River, McLennan County to Fort Bend County, Texas, March 6-10, 2006

8. Streamflow gains and losses computed for 27 reaches on the Brazos River, McLennan County to Fort Bend County, Texas, August 10-18, 2006 


\section{Conversion Factors and Datum}

\section{Inch/Pound to SI}

\begin{tabular}{|c|c|c|}
\hline Multiply & By & To obtain \\
\hline \multicolumn{3}{|c|}{ Length } \\
\hline foot $(\mathrm{ft})$ & 0.3048 & meter $(\mathrm{m})$ \\
\hline inch (in.) & 25.4 & millimeter $(\mathrm{mm})$ \\
\hline mile (mi) & 1.609 & kilometer $(\mathrm{km})$ \\
\hline \multicolumn{3}{|c|}{ Area } \\
\hline square mile $\left(\mathrm{mi}^{2}\right)$ & 2.590 & square kilometer $\left(\mathrm{km}^{2}\right)$ \\
\hline \multicolumn{3}{|c|}{ Flow } \\
\hline cubic feet per second $\left(\mathrm{ft}^{3} / \mathrm{s}\right)$ & 0.02832 & cubic meters per second $\left(\mathrm{m}^{3} / \mathrm{s}\right)$ \\
\hline
\end{tabular}

Temperature in degrees Celsius $\left({ }^{\circ} \mathrm{C}\right)$ may be converted to degrees Fahrenheit $\left({ }^{\circ} \mathrm{F}\right)$ as follows:

${ }^{\circ} \mathrm{F}=\left(1.8 x^{\circ} \mathrm{C}\right)+32$

Specific conductance is given in microsiemens per centimeter at 25 degrees Celsius $(\mu \mathrm{S} / \mathrm{cm})$.

Water year is defined as the 12-month period October 1 through September 30 and is designated by the calendar year in which it ends.

\section{Datum}

Horizontal coordinate information is referenced to the North American Datum of 1983 (NAD 83). 
Blank Page 


\title{
Base Flow (1966-2005) and Streamflow Gain and Loss (2006) of the Brazos River, McLennan County to Fort Bend County, Texas, 2006
}

\author{
By Michael J. Turco, Jeffery W. East, and Matthew S. Milburn
}

\section{Abstract}

During 2006-07, the U.S. Geological Survey (USGS), in cooperation with the Texas Water Development Board, did a study to quantify historical (water years 1966-2005) base flow and streamflow gains and losses from two streamflowmeasuring surveys (March and August 2006) in the Brazos River from McLennan County to Fort Bend County, Texas. The Brazos River is hydraulically connected to the Brazos River alluvium aquifer, which in turn is hydraulically connected to several underlying aquifers, the outcrops of which occur in laterally adjacent layers generally parallel to the coast (major aquifers, Carrizo-Wilcox and Gulf Coast, and minor aquifers, Queen City, Sparta, and Yegua-Jackson). Hydrograph separation was done using the USGS computer program Hydrograph Separation and Analysis with historical streamflow from 10 USGS gaging stations, three on the Brazos River and seven on selected tributaries to the Brazos River. Streamflow data for computation of gains and losses were collected in March 2006 from 36 sites on the Brazos River and 19 sites on 19 tributaries to the Brazos River; and in August 2006 from 28 sites on the Brazos River and 16 sites on tributaries. Hydrograph separation and associated analyses indicate an appreciable increase in base flow as a percentage of streamflow in the reach of the Brazos River that crosses the outcrops of the Carrizo-Wilcox, Queen City, Sparta, and Yegua-Jackson aquifers compared to that in the adjacent upstream reach (on average from about 43 percent to about 60 percent). No increase in base flow as a percentage of streamflow in the reach of the Brazos River crossing the Gulf Coast aquifer compared to that in the adjacent upstream reach was indicated. Streamflow gains and losses computed for March 2006 for 35 reaches defined by pairs of sites on the Brazos River indicated that five reaches were verifiably gaining streamflow (computed gain exceeded potential flow measurement error) and none were verifiably losing streamflow. Four of the five gaining reaches are in the outcrop areas of the Carrizo-Wilcox and Yegua-Jackson aquifers. The results of the synoptic gain and loss surveys are consistent with the results of the base- flow analysis of historical streamflow. Appreciable increases in streamflow, apparently the result of increases in base flow, occur in the reach of the Brazos River that crosses the outcrops of the Carrizo-Wilcox, Queen City, Sparta, and Yegua-Jackson aquifers.

\section{Introduction}

The Brazos River alluvium aquifer is an important resource to water users in the Brazos River Basin from McLennan County to Fort Bend County, Texas (fig. 1). As demands on the resource increase in the future, the Brazos River alluvium aquifer likely will become more important. Quantification of base flow in the Brazos River and the exchange of water between the Brazos River and the underlying Brazos River alluvium aquifer could assist water managers and planners responsible for the long-term sustainability of surface- and ground-water resources in the area.

Slade and others (2002) identified 31 previous studies in which streamflow gain and loss data were collected in the Brazos River, which extends across Texas from southeastern New Mexico to the Gulf of Mexico (fig. 1). However, none of those studies were done in the reach of the river that flows through the area of the Brazos River alluvium aquifer (the reach that defines the area of this study). General qualitative descriptions of base flow in segments of the study area were reported by the Texas Board of Water Engineers (1960):

- Area between Waco and the northern Falls County line-Geologic formations can be expected to make extremely small contributions to base flow of the Brazos River.

- Area between the northern Falls County line and Bryan-Geologic formations can be expected to make large contributions to base flow of the Brazos River.

- Area between Bryan and Hempstead-Geologic formations can be expected to make small contributions to base flow of the Brazos River. 


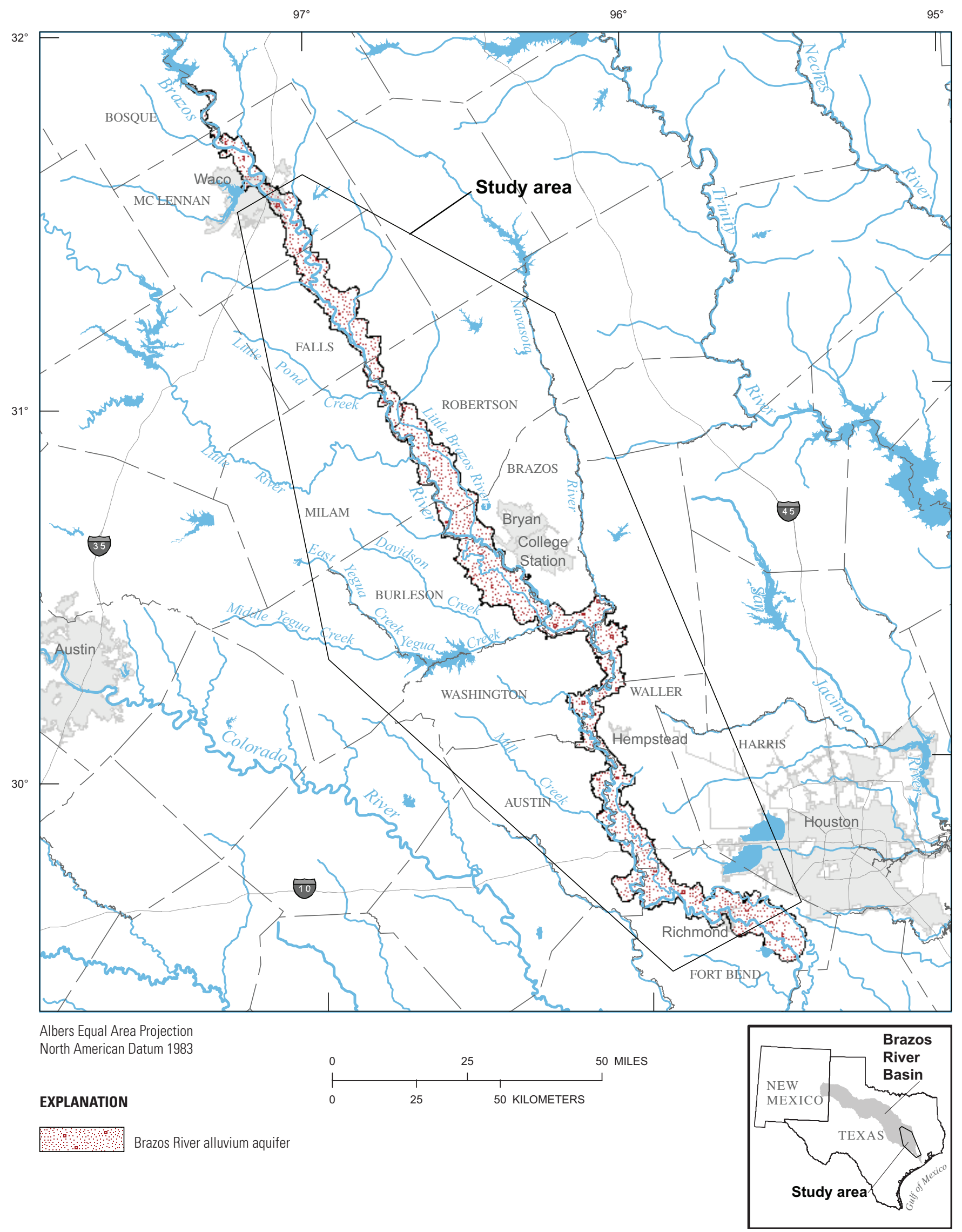

Figure 1. Study area, Brazos River, and extent of Brazos River alluvium aquifer, McLennan County to Fort Bend County, Texas. 
- Area between Hempstead and Richmond-Geologic formations can be expected to make moderate contributions to base flow of the Brazos River.

During 2006-07, the U.S. Geological Survey (USGS), in cooperation with the Texas Water Development Board (TWDB), did a study to quantify base flow and streamflow gains and losses in the Brazos River from McLennan County to Fort Bend County. Of particular interest were potential gains and losses in the river where it flows across the outcrop areas of the Carrizo-Wilcox aquifer, the Queen City aquifer, the Sparta aquifer, and the Yegua-Jackson aquifer (fig. 2). These outcrop areas are essentially coincident with the area between the southern Falls County line and Bryan that, according to the Texas Board of Water Engineers (1960), is expected to make large contributions to base flow of the Brazos River.

\section{Purpose and Scope}

The purpose of this report is to document the results of base-flow analysis (hydrograph separation) of historical (water years 1966-2005 [October 1965 through September 2005]) streamflow and two synoptic streamflow gain and loss surveys of the Brazos River from McLennan County to Fort Bend County, Texas, in March and August 2006. Methods of data collection and analysis are described. Results of hydrograph separation based on available historical data for three sites on the Brazos River and seven sites on tributaries to the Brazos River are presented. Streamflow gains and losses based on flow data collected from 55 sites on the Brazos River and selected tributaries during the two synoptic surveys are presented, and the respective gaining and losing reaches are identified. Water-quality properties (temperature and specific conductance) were collected as a part of the March 2006 survey and are included in the report, but those data were not used in the analysis of gains and losses.

\section{Description of Study Area}

The study area encompasses a 310-mile reach of the Brazos River from Waco in McLennan County to Richmond in Fort Bend County (fig. 1) and includes selected inflow tributaries along the reach. The major tributaries are the Little River, Little Brazos River, Yegua Creek, Navasota River, and Mill Creek. Average annual rainfall (1971-2000) at College Station is 39.67 inches (National Weather Service, 2007). Five real-time USGS streamflow-gaging stations are on the Brazos River in the study area (fig. 2):

1. 08096500 Brazos River at Waco, Tex. (site B1, fig. 2)

2. 08098290 Brazos River near Highbank, Tex. (site B6, fig. 2)

3. 08108700 Brazos River at State Highway 21 near Bryan, Tex. (site B14, fig. 2)
4. 08111500 Brazos River near Hempstead, Tex. (site B26, fig. 2)

5. 08114000 Brazos River at Richmond, Tex. (site B36, fig. 2)

Daily mean streamflow (1941-2006) ranged from 2,147 cubic feet per second at the Brazos River at Waco to 7,532 cubic feet per second at the Brazos River at Richmond (U.S. Geological Survey, 2007).

The Brazos River is hydraulically connected to the Brazos River alluvium aquifer. The alluvium aquifer is composed of fine to coarse sand, gravel, silt, and clay, the distribution of which varies from place to place (Cronin and others, 1963).

Beds or lenses of sand and gravel grade laterally into vertically finer or coarser material. In general, the finer material is in the upper part of the aquifer. The thickness of the aquifer throughout most of its extent is less than 100 feet (Shah and others, 2007, sheet 4).

Underlying the Brazos River alluvium aquifer in laterally adjacent layers generally parallel to the coast are two major aquifers, Carrizo-Wilcox and Gulf Coast, and three minor aquifers, Queen City, Sparta, and Yegua-Jackson (Texas Water Development Board, 2007) (fig. 2). The Gulf Coast aquifer is actually an aquifer system, the principal aquifers of which are the Chicot, Evangeline, and Jasper (not differentiated in this report). Upstream from the outcrop of the Carrizo-Wilcox aquifer, the Brazos River alluvium aquifer is underlain by rocks not characterized as part of a major or minor aquifer by the TWDB. Like the Brazos River alluvium aquifer, each of the underlying aquifers is composed of interbedded sand, clay, gravel, and silt in varying amounts (Ashworth and Hopkins, 1995; Ryder and Ardis, 2002). The underlying aquifers are distinguished from one another in large part by the stratigraphic units of the rocks composing the aquifers and the relative positions of the units.

The underlying aquifers are hydraulically connected to the Brazos River alluvium aquifer, as indicated by potentiometric-surface maps of the Carrizo-Wilcox, Queen City, and Sparta aquifers (Garza and others, 1987). The approximate potentiometric-surface map of the Carrizo-Wilcox aquifer for 1980 of Garza and others (1987) in the aquifer outcrop shows U-shaped potentiometric contours, closed upstream and relatively large, across the Brazos River. Similarly, the approximate potentiometric-surface map of the Sparta-Queen City aquifer (Garza and others [1987] combined the Queen City and Sparta aquifers for mapping) for 1980 in the aquifer outcrop shows U-shaped potentiometric contours, closed upstream, across the Brazos River. The potentiometric-surface maps indicate, in addition to hydraulic connection between the Brazos River, the Brazos River alluvium aquifer, and the underlying aquifers, that the river is gaining water in the aquifer outcrop areas, which is consistent with the Texas Board of Water Engineers (1960) qualitative description of the river gaining base flow in the reaches crossing the aquifer outcrops. Although no potentiometric surface for the Yegua-Jackson aquifer is available, and no potentiometric-surface maps 


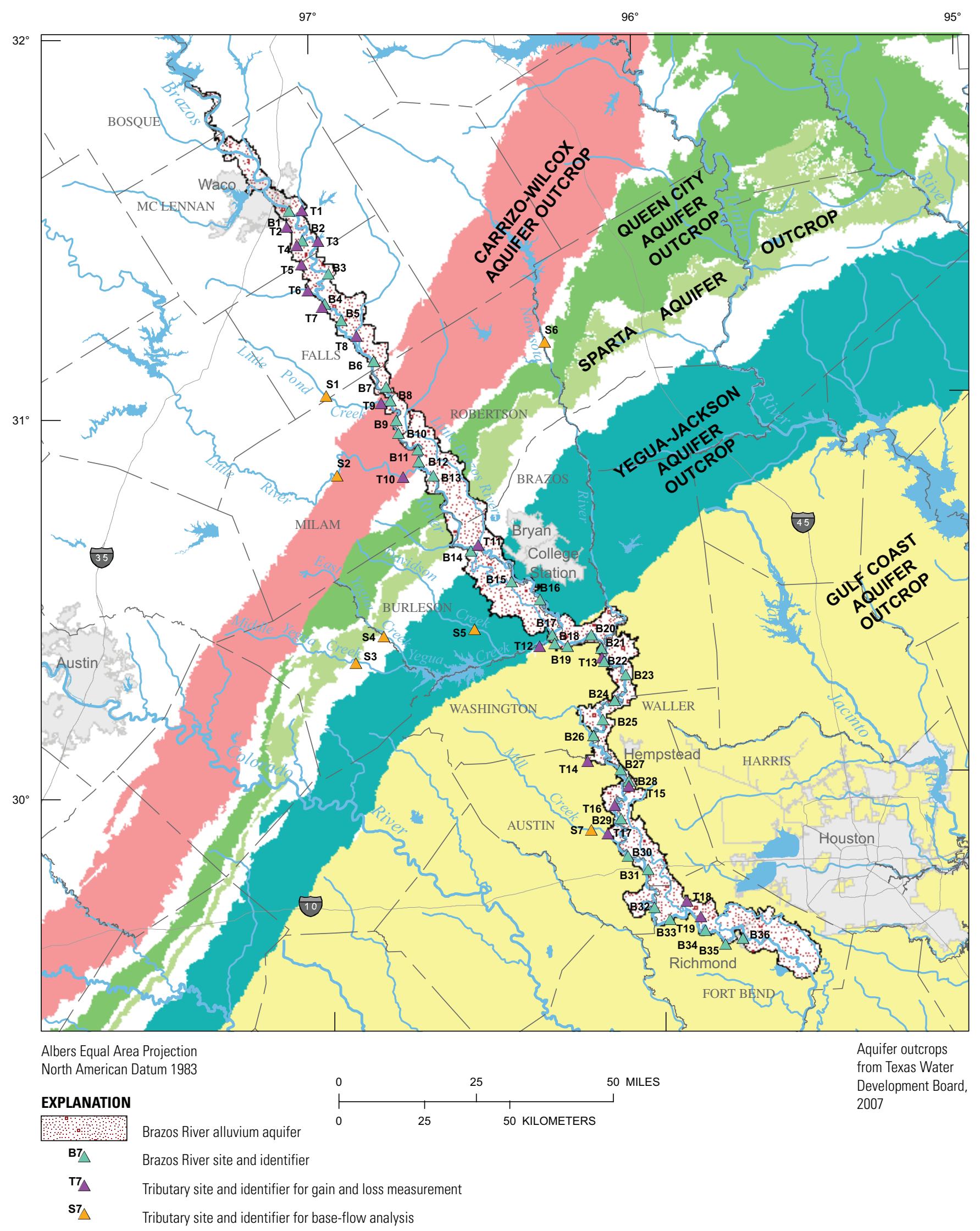

Figure 2. Brazos River alluvium aquifer, McLennan County to Fort Bend County, Texas; outcrops of underlying aquifers; and datacollection sites. 
constructed using shallow wells in the outcrops of the Chicot, Evangeline, and Jasper aquifers are known to exist, hydraulic connection between those aquifers, the Brazos River alluvium aquifer, and Brazos River are assumed.

\section{Acknowledgments}

The authors acknowledge the contributions of Barney Austin, Robert Mace, and Ali Chowdhury of the Texas Water Development Board during the planning of this study. Additionally, the authors acknowledge staff of the Brazos River Authority who assisted in identifying appropriate streamflowmeasuring sites and in collecting data. The authors thank the numerous property owners who allowed access to measuring sites.

\section{Methods}

Hydrograph separation using historical streamflow was done to estimate base flow. Streamflow data collection and streamflow gain and loss computation were done to estimate streamflow gains and losses in the reach of the Brazos River from McLennan County to Fort Bend County.

\section{Hydrograph Separation}

Hydrograph separation is the process of separating measured streamflow into components resulting either from direct surface runoff or from ground-water discharge (base flow). Hydrograph separation was done using the USGS computer program Hydrograph Separation and Analysis (HYSEP) (Sloto and Crouse, 1996). The data used in HYSEP were historical (water years 1966-2005) streamflow from 10 USGS gaging stations, three on the Brazos River and seven on selected tributaries to the Brazos River (table 1). The HYSEP program makes the hydrograph separation process objective by removing inconsistencies inherent in manual methods. Daily mean streamflow for each station was input to the HYSEP program. Output from the program includes the two components of the annual mean streamflow - annual mean surface runoff and annual mean base flow. Additionally, HYSEP provides the base-flow index (BFI), which is the ratio of the annual mean base flow to the annual mean streamflow. The BFI was used as a means to graphically compare streamflows (hydrographs) from the three Brazos River stations. The graphical comparisons indicate whether the reach between two stations is gaining or losing flow.

Wahl and Wahl (1995) states that caution should be used in applying hydrograph separation to data for short-term storms or for sites where streamflow is affected by upstream regulation, such as reservoir releases. The Brazos River in the study area is affected by regulation. Therefore, after using HYSEP to make hydrograph separation computations, the results were analyzed and judgments made regarding whether rises were from storm runoff or from reservoir releases. When judged necessary, HYSEP program input was adjusted by varying the runoff-timing parameter, and the program was rerun. This iterative process was continued until the results were deemed reasonable (that is, periods of reservoir releases were categorized by HYSEP as storm runoff).

\section{Streamflow Data Collection}

Streamflow data for computation of gains and losses were collected from the Brazos River and 19 tributaries to the Brazos River during March 6-10, 2006, and during August 10-18, 2006. The study was separated into two periods to collect streamflow data that were representative of two distinct hydrologic conditions. March is usually wetter than August and demand on water resources (pumpage from the river, the alluvium aquifer, and underlying major and minor aquifers) is relatively low. August is usually drier and demand on water resources is relatively high.

During the March data-collection period, streamflow was measured at 55 sites, 36 on the Brazos River and 19 on tributaries (fig. 2; table 2). During the August data-collection period, streamflow measurements were made at 44 of the 55 sites, 28 on the Brazos River and 16 on tributaries. Water temperature and specific conductance also were measured during the March data-collection period at 53 and 52 sites, respectively.

Table 1. U.S. Geological Survey streamflow-gaging stations on the Brazos River and selected tributaries, McLennan County to Fort Bend County, Texas, that provided data for hydrograph separation, water years 1966-2005 (October 1965 through September 2005).

[USGS, U.S. Geological Survey]

\begin{tabular}{|c|c|c|c|}
\hline $\begin{array}{c}\text { Site } \\
\text { identi- } \\
\text { fier } \\
\text { (fig. 2) }\end{array}$ & $\begin{array}{c}\text { USGS } \\
\text { station } \\
\text { number }\end{array}$ & USGS station name & $\begin{array}{l}\text { Contri- } \\
\text { buting } \\
\text { drainage } \\
\text { area } \\
\text { (square } \\
\text { miles) }\end{array}$ \\
\hline B6 & 08098290 & Brazos River near Highbank, Tex. & 20,870 \\
\hline $\mathrm{S} 1$ & 08098300 & $\begin{array}{l}\text { Little Pond Creek near } \\
\text { Burlington, Tex. }\end{array}$ & 23.0 \\
\hline $\mathrm{S} 2$ & 08106500 & Little River near Cameron, Tex. & 7,065 \\
\hline S3 & 08109700 & $\begin{array}{l}\text { Middle Yegua Creek near Dime Box, } \\
\text { Tex. }\end{array}$ & 236 \\
\hline S4 & 08109800 & East Yegua Creek near Dime Box, Tex. & 244 \\
\hline S5 & 08110100 & Davidson Creek near Lyons, Tex. & 195 \\
\hline S6 & 08110500 & Navasota River near Easterly, Tex. & 968 \\
\hline B26 & 08111500 & Brazos River near Hempstead, Tex. & 34,314 \\
\hline S7 & 08111700 & Mill Creek near Bellville, Tex. & 376 \\
\hline B36 & 08114000 & Brazos River at Richmond, Tex. & 35,541 \\
\hline
\end{tabular}


Table 2. Sites on the Brazos River and selected tributaries, McLennan County to Fort Bend County, Texas, at which measurements for streamflow gain and loss computation were made, March and August 2006.

[USGS, U.S. Geological Survey; SH, State Highway; --, not applicable; CR, County Road, FM, Farm Road; IH, Interstate Highway]

\begin{tabular}{|c|c|c|c|c|c|}
\hline $\begin{array}{c}\text { Site } \\
\text { identifier } \\
\text { (fig. 2) }\end{array}$ & $\begin{array}{c}\text { USGS } \\
\text { station number }\end{array}$ & USGS station name & $\begin{array}{l}\text { Latitude } \\
\text { (degrees } \\
\text { minutes } \\
\text { seconds) }\end{array}$ & $\begin{array}{c}\text { Longitude } \\
\text { (degrees } \\
\text { minutes } \\
\text { seconds) }\end{array}$ & $\begin{array}{l}\text { Distance } \\
\text { upstream } \\
\text { from mouth } \\
\text { (miles) }\end{array}$ \\
\hline B1 & 08096500 & Brazos River at Waco, Tex. & 313209 & 0970423 & 401 \\
\hline $\mathrm{T} 1$ & 313210097015800 & Tehuacana Creek at SH 6 near Waco, Tex. & 313210 & 0970158 & -- \\
\hline $\mathrm{T} 2$ & 312934097045300 & Flat Creek at CR 3400 near Robinson, Tex. & 312934 & 0970453 & -- \\
\hline $\mathrm{T} 3$ & 312715096590800 & Monas Creek at FM 1860 near Riesel, Tex. & 312715 & 0965908 & -- \\
\hline $\mathrm{T} 4$ & 312639097030500 & Castleman Creek near Robinson, Tex. & 312639 & 0970305 & -- \\
\hline T5 & 312335097022100 & Bull Hide Creek at FM 434 near Golinda, Tex. & 312335 & 0970221 & -- \\
\hline B3 & 312201096572200 & Brazos River near Golinda, Tex. & 312201 & 0965722 & 375 \\
\hline T6 & 311927097011700 & Cow Bayou below FM 434 near Chilton, Tex. & 311927 & 0970117 & -- \\
\hline $\mathrm{T} 8$ & 311204096523300 & Mussel Run Creek near Highbank, Tex. & 311204 & 0965233 & -- \\
\hline B6 & 08098290 & Brazos River near Highbank, Tex. & 310802 & 0964929 & 346 \\
\hline B7 & 310354096472000 & Brazos River below FM 1373 near Baileyville, Tex. & 310354 & 0964720 & 338 \\
\hline B8 & 310143096463800 & Brazos River above Pond Creek near Baileyville, Tex. & 310143 & 0964638 & 335 \\
\hline T9 & 310120096481700 & Pond Creek at FM 2027 near Baileyville, Tex. & 310120 & 0964817 & -- \\
\hline B9 & 305836096453800 & Brazos River at FM 979 near Calvert, Tex. & 305836 & 0964538 & 329 \\
\hline B10 & 305630096451400 & Brazos River near Calvert, Tex. & 305630 & 0964514 & 326 \\
\hline B11 & 305349096414800 & Brazos River at Big Bend near Calvert, Tex. & 305349 & 0964148 & 317 \\
\hline B12 & 305158096414500 & Brazos River at FM 485 near Hearne, Tex. & 305158 & 0964145 & 314 \\
\hline $\mathrm{T} 10$ & 304932096443900 & Little River at CR 263 near Gause, Tex. & 304932 & 0964439 & -- \\
\hline $\mathrm{T} 12$ & 302208096203500 & Yegua Creek at FM 50 near Clay, Tex. & 302208 & 0962035 & -- \\
\hline B18 & 302230096175000 & Brazos River near FM 1955 near Clay, Tex. & 302230 & 0961750 & 241 \\
\hline B19 & 302200096152900 & Brazos River at Rogers Plantation near Millican, Tex. & 302200 & 0961529 & 239 \\
\hline $\mathrm{B} 20$ & 302342096110400 & Brazos River near FM 159 near Millican, Tex. & 302342 & 0961104 & 233 \\
\hline B21 & 302134096091800 & Brazos River at SH 105 near Washington, Tex. & 302134 & 0960918 & 228 \\
\hline T13 & 302003096091400 & Navasota River below SH 105 near Washington, Tex. & 302003 & 0960914 & -- \\
\hline B22 & 301927096085300 & Brazos River below Navasota River near Washington, Tex. & 301927 & 0960853 & 226 \\
\hline B23 & 301713096050000 & Brazos River at Old River Road near Courtney, Tex. & 301713 & 0960500 & 219 \\
\hline B24 & 301313096071200 & Brazos River near FM 2726 near Courtney, Tex. & 301313 & 0960712 & 209 \\
\hline B25 & 301014096092700 & Brazos River near FM 1736 near Hempstead, Tex. & 301014 & 0960927 & 202 \\
\hline B26 & 08111500 & Brazos River near Hempstead, Tex. & 300744 & 0961115 & 194 \\
\hline T14 & 300343096123300 & Caney Creek at FM 1371 near Hempstead, Tex. & 300343 & 0961233 & -- \\
\hline $\mathrm{B} 27$ & 300217096063400 & Brazos River at SH 159 near Hempstead, Tex. & 300217 & 0960634 & 179 \\
\hline B28 & 300024096050500 & Brazos River near FM 1887 near Hempstead, Tex. & 300024 & 0960505 & 176 \\
\hline $\mathrm{T} 15$ & 295936096051100 & Clear Creek near FM 1887 near Hempstead, Tex. & 295936 & 0960511 & -- \\
\hline T16 & 295637096075400 & Piney Creek at FM 331 near Burleigh, Tex. & 295637 & 0960754 & -- \\
\hline
\end{tabular}


Table 2. Sites on the Brazos River and selected tributaries, McLennan County to Fort Bend County, Texas, at which measurements for streamflow gain and loss computation were made, March and August 2006 — Continued.

\begin{tabular}{|c|c|c|c|c|c|}
\hline $\begin{array}{c}\text { Site } \\
\text { identifier } \\
\text { (fig. 2) }\end{array}$ & $\begin{array}{c}\text { USGS } \\
\text { station number }\end{array}$ & USGS station name & $\begin{array}{l}\text { Latitude } \\
\text { (degrees } \\
\text { minutes } \\
\text { seconds) }\end{array}$ & $\begin{array}{l}\text { Longitude } \\
\text { (degrees } \\
\text { minutes } \\
\text { seconds) }\end{array}$ & $\begin{array}{c}\text { Distance } \\
\text { upstream } \\
\text { from mouth } \\
\text { (miles) }\end{array}$ \\
\hline B29 & 295431096064800 & Brazos River at FM 529 near Burleigh, Tex. & 295431 & 0960648 & 162 \\
\hline $\mathrm{T} 17$ & 295211096091900 & Mill Creek at FM 331 near Burleigh, Tex. & 295211 & 0960919 & -- \\
\hline B30 & 294830096054400 & Brazos River at FM 1458 at San Felipe, Tex. & 294830 & 0960544 & 147 \\
\hline B32 & 294017096011400 & Brazos River at FM 1093 at Simonton, Tex. & 294017 & 0960114 & 125 \\
\hline B33 & 293820095583200 & Brazos River at FM 1489 near Simonton, Tex. & 293820 & 0955832 & 117 \\
\hline $\mathrm{T} 18$ & 294107095551800 & Bessies Creek at FM 1093 near Fulshear, Tex. & 294107 & 0955518 & -- \\
\hline T19 & 293835095525600 & Jones Creek at Bois du Arc Road near Fulshear, Tex. & 293835 & 0955256 & -- \\
\hline B34 & 293621095521300 & Brazos River at CR near FM 359 near Rosenberg, Tex. & 293621 & 0955213 & 103 \\
\hline
\end{tabular}

Discrete measurements of streamflow were made using USGS methods described in Rantz and others (1982). Where conditions allowed (water depths generally less than 3 feet), streams were waded and velocity measurements were made using rod-mounted acoustic meters (Sontek/YSI, Inc., 2007). In all other instances, boat-mounted acoustic Doppler current profilers were used to measure streamflow, as described in Oberg and others (2005). In-place measurements of water temperature and specific conductance were made using methods described by Wilde and Radtke (1998).

\section{Streamflow Gain and Loss Computation}

Streamflow gains and losses over the course of a stream are measured indirectly by computing the differences in streamflow between sites along the stream. Sources of gains or losses between two sites (which define a reach), in addition to ground-water inflow or outflow through the streambed, include tributary inflow, diversions, return flows, and evaporation. Using these factors, streamflow gain or loss in the reach is computed as

$$
\mathrm{G}=\mathrm{Q}_{\mathrm{D}}-\mathrm{Q}_{\mathrm{U}}-\mathrm{I}+\mathrm{D}-\mathrm{R}+\mathrm{E},
$$

where

$\mathrm{G}=$ streamflow gain or loss (ground-water inflow or outflow);

$\mathrm{Q}_{\mathrm{D}}=$ measured streamflow at the downstream boundary of the reach;

$\mathrm{Q}_{\mathrm{U}}=$ measured streamflow at the upstream boundary of the reach;

$I=$ measured inflows from tributaries;

$\mathrm{D}=$ measured outflows (diversions) from the reach;

$\mathrm{R}=$ return flows to the reach; and

$\mathrm{E}=$ evaporation. (Units of all variables are cubic feet per second.)
During this study, numerous tributary inflows and one diversion were measured directly. However, return flows and evaporation were not measured, and those components were excluded from equation 1 . The magnitude of error associated with these exclusions is believed to be minor when compared to potential errors associated with the flow measurements.

For this report, a stream reach is classified as verifiably gaining or losing only when the difference between streamflows at the upstream and downstream measuring sites that define the reach is greater than the potential error associated with the flow measurements. Measurement error is based on the rating of the streamflow measurement (excellent, good, fair, or poor) by the streamgager (Sauer and Meyer, 1992). The rating is based on factors such as cross-section uniformity, velocity homogeneity, streambed conditions, and other factors that affect the accuracy of the measurement. Measurements rated excellent are believed to be within 2 percent of the actual flow, good are believed to be within 5 percent, fair are believed to be within 8 percent, and poor are believed to differ from the actual flow by more than 8 percent. The potential errors associated with each pair of streamflow measurements for a given reach were summed to obtain the potential composite error for comparison with the computed gain or loss.

\section{Base Flow}

The results of hydrograph separation for three streamflow-gaging stations on the Brazos River, 08098290 Brazos River near Highbank (site B6, fig. 2), 08111500 Brazos River near Hempstead (site B26, fig. 2), and 08114000 Brazos River at Richmond (site B36, fig. 2) for water years 1966-2005 are shown in figure 3 and listed in table 3. At the Highbank station, base flow as a percentage of streamflow ranged from a high of about 59 percent in 1990 to a low of about 30 percent 
A. 08098290 Brazos River near Highbank, Texas

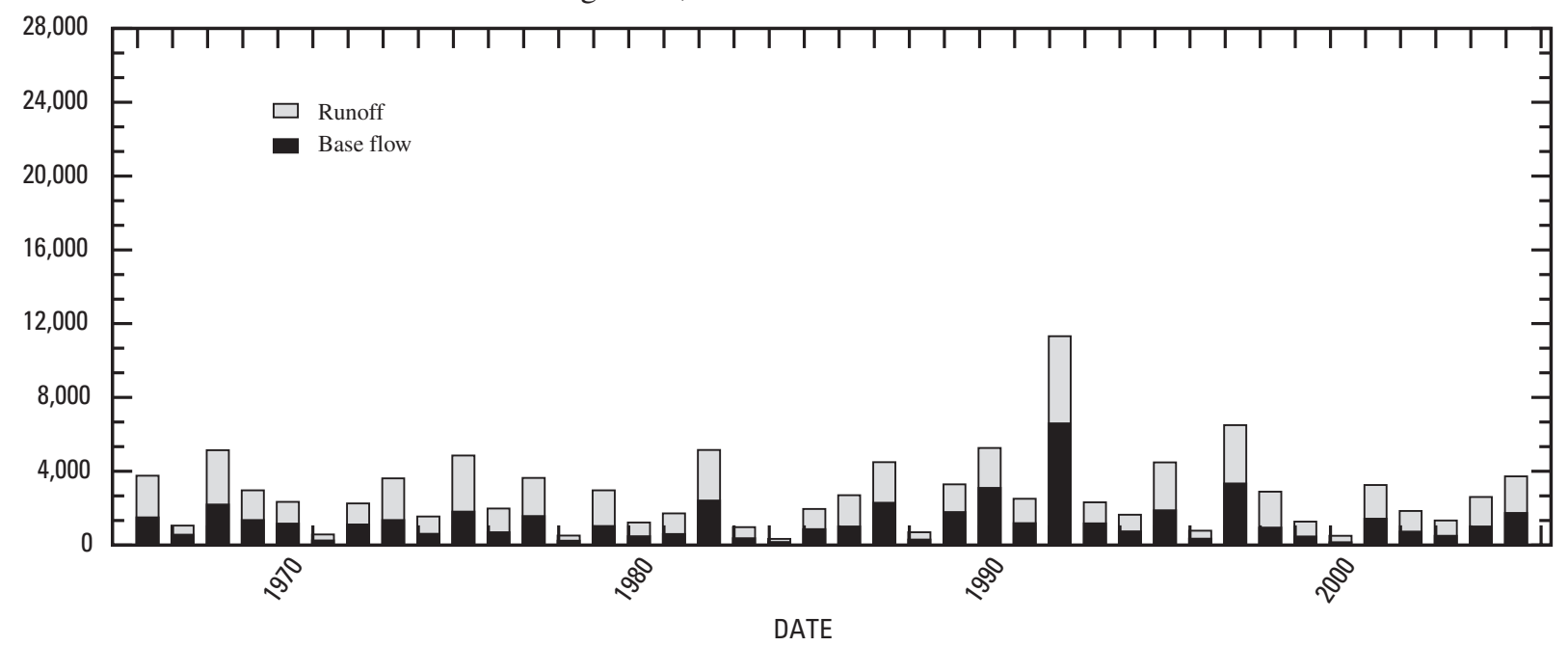

B. 08111500 Brazos River near Hempstead, Texas

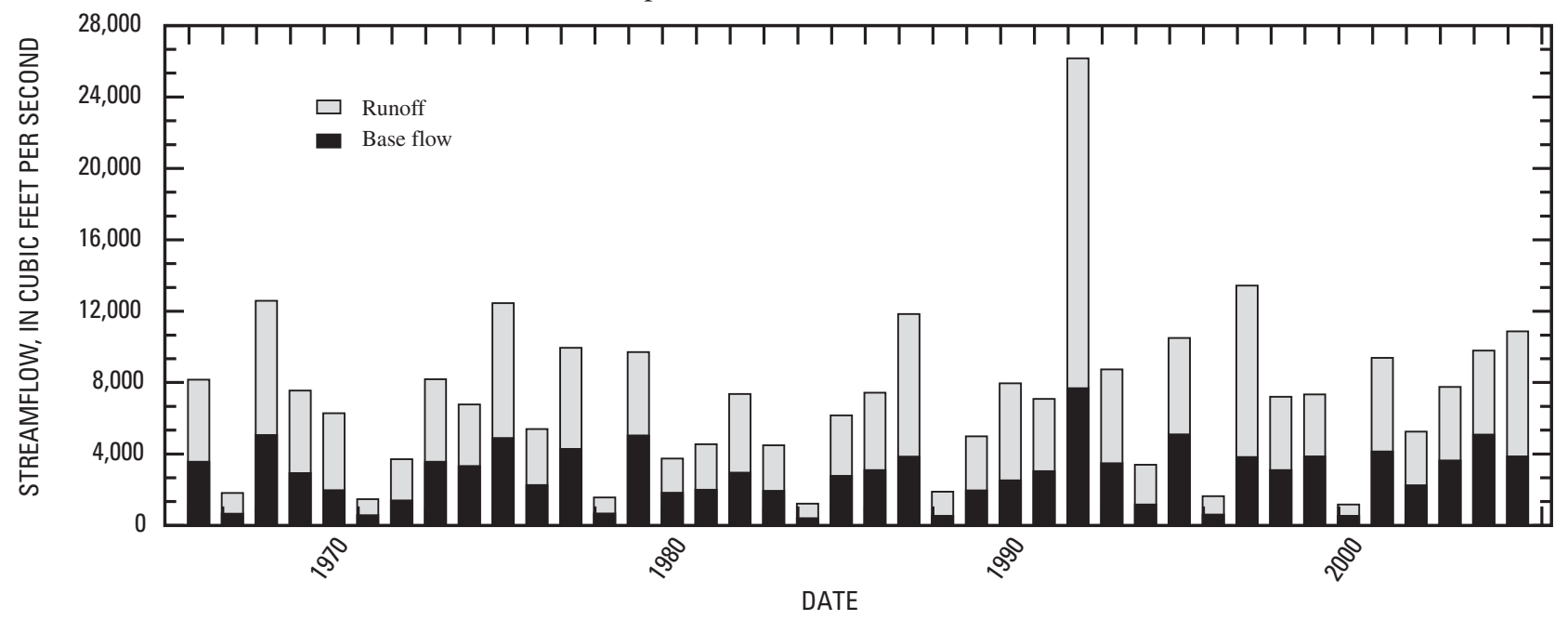

C. 08114000 Brazos River at Richmond, Texas.

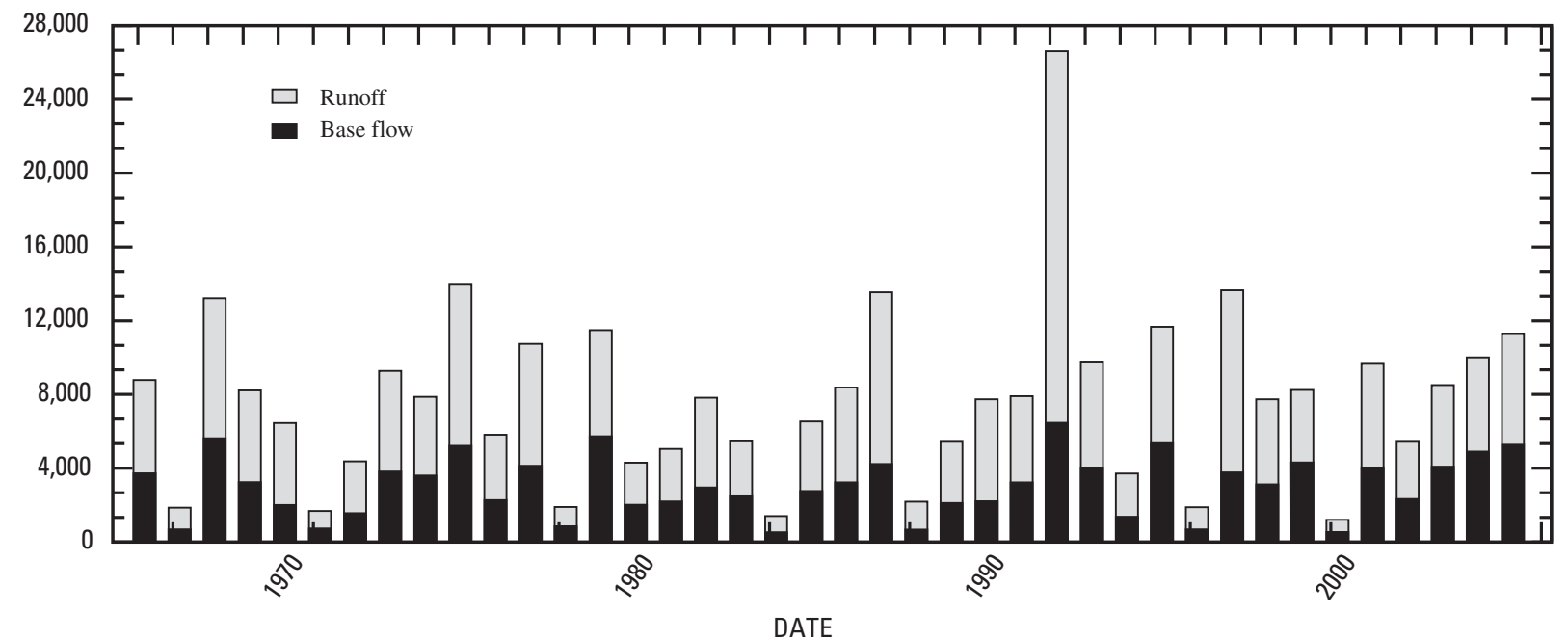

Figure 3. Base flow and runoff components of streamflow, 1966-2005, from hydrograph separation for (A) 08098290 Brazos River near Highbank, Texas; (B) 08111500 Brazos River near Hempstead, Texas; and (C) 08114000 Brazos River at Richmond, Texas. 
Table 3. Results of hydrograph separation for 08098290 Brazos River near Highbank, Texas, 08111500 Brazos River near Hempstead, Texas, and 08114000 Brazos River at Richmond, Texas, 1966-2005.

[Q, annual mean streamflow; ft³/s, cubic feet per second; BF, base-flow component; RO, runoff component; BFI, base-flow index (defined as BF/Q)]

\begin{tabular}{|c|c|c|c|c|c|c|c|c|c|c|c|c|}
\hline \multirow{2}{*}{$\begin{array}{l}\text { Water } \\
\text { year }\end{array}$} & \multicolumn{4}{|c|}{$\begin{array}{c}08098290 \text { Brazos River near } \\
\text { Highbank, Tex. }\end{array}$} & \multicolumn{4}{|c|}{$\begin{array}{c}08111500 \text { Brazos River near } \\
\text { Hempstead, Tex. }\end{array}$} & \multicolumn{4}{|c|}{$\begin{array}{l}08114000 \text { Brazos River at } \\
\text { Richmond, Tex. }\end{array}$} \\
\hline & $\underset{\left(f t^{3} / s\right)}{0}$ & $\begin{array}{c}\mathrm{BF} \\
\left(\mathrm{ft}^{3} / \mathbf{s}\right)\end{array}$ & $\begin{array}{c}\text { Ro } \\
\left(\mathrm{ft}^{3} / \mathbf{s}\right)\end{array}$ & BFI & $\begin{array}{c}0 \\
\left(\mathrm{ft}^{3} / \mathrm{s}\right)\end{array}$ & $\begin{array}{c}\mathrm{BF} \\
\left(\mathrm{ft}^{3} / \mathbf{s}\right)\end{array}$ & $\begin{array}{c}\mathrm{RO} \\
\left(\mathrm{ft}^{3} / \mathrm{s}\right)\end{array}$ & BFI & $\underset{\left(\mathrm{ft}^{\mathrm{t}} / \mathrm{s}\right)}{\mathbf{0}}$ & $\begin{array}{c}\mathrm{BF} \\
\left(\mathrm{ft}^{\mathrm{t}} / \mathrm{s}\right)\end{array}$ & $\begin{array}{c}\text { RO } \\
\left(\mathrm{ft}^{3} / \mathrm{s}\right)\end{array}$ & BFI \\
\hline 1966 & 3,759 & 1,503 & 2,255 & 0.40 & 8,156 & 4,596 & 3,560 & 0.56 & 8,792 & 5,066 & 3,726 & 0.58 \\
\hline 1967 & 1,051 & 565 & 485 & .54 & 1,822 & 1,164 & 658 & .64 & 1,866 & 1,187 & 679 & .63 \\
\hline 1968 & 5,144 & 2,195 & 2,950 & .43 & 12,593 & 7,534 & 5,059 & .60 & 13,226 & 7,597 & 5,629 & .57 \\
\hline 1969 & 2,963 & 1,354 & 1,610 & .46 & 7,546 & 4,619 & 2,927 & .61 & 8,218 & 4,974 & 3,245 & .60 \\
\hline 1970 & 2,337 & 1,161 & 1,176 & .50 & 6,286 & 4,312 & 1,973 & .69 & 6,459 & 4,453 & 2,006 & .68 \\
\hline 1971 & 572 & 244 & 328 & .43 & 1,466 & 893 & 573 & .61 & 1,678 & 941 & 737 & .56 \\
\hline 1972 & 2,253 & 1,109 & 1,144 & .49 & 3,703 & 2,308 & 1,396 & .62 & 4,374 & 2,807 & 1,566 & .64 \\
\hline 1973 & 3,611 & 1,355 & 2,257 & .38 & 8,186 & 4,616 & 3,570 & .56 & 9,282 & 5,464 & 3,818 & .59 \\
\hline 1974 & 1,552 & 611 & 941 & .39 & 6,776 & 3,447 & 3,329 & .51 & 7,876 & 4,266 & 3,611 & .54 \\
\hline 1975 & 4,853 & 1,815 & 3,038 & .37 & 12,452 & 7,560 & 4,829 & .61 & 13,956 & 8,735 & 5,221 & .63 \\
\hline 1976 & 1,980 & 693 & 1,287 & .35 & 5,400 & 3,137 & 2,263 & .58 & 5,817 & 3,540 & 2,277 & .61 \\
\hline 1977 & 3,643 & 1,568 & 2,075 & .43 & 9,958 & 5,674 & 4,284 & .57 & 10,740 & 6,601 & 4,139 & .61 \\
\hline 1978 & 517 & 233 & 284 & .45 & 1,564 & 898 & 666 & .57 & 1,894 & 1,038 & 856 & .55 \\
\hline 1979 & 2,970 & 1,031 & 1,940 & .35 & 9,722 & 4,691 & 5,031 & .48 & 11,487 & 5,749 & 5,738 & .50 \\
\hline 1980 & 1,218 & 474 & 744 & .39 & 3,741 & 1,917 & 1,823 & .51 & 4,307 & 2,284 & 2,023 & .53 \\
\hline 1981 & 1,711 & 593 & 1,118 & .35 & 4,541 & 2,546 & 1,995 & .56 & 5,053 & 2,852 & 2,201 & .56 \\
\hline 1982 & 5,150 & 2,414 & 2,736 & .47 & 7,360 & 4,403 & 2,957 & .60 & 7,829 & 4,871 & 2,958 & .62 \\
\hline 1983 & 970 & 363 & 607 & .37 & 4,491 & 2,551 & 1,940 & .57 & 5,462 & 2,982 & 2,480 & .54 \\
\hline 1984 & 329 & 162 & 167 & .49 & 1,216 & 817 & 399 & .67 & 1,403 & 881 & 522 & .63 \\
\hline 1985 & 1,959 & 865 & 1,094 & .44 & 6,159 & 3,384 & 2,775 & .55 & 6,540 & 3,775 & 2,765 & .58 \\
\hline 1986 & 2,697 & 1,002 & 1,695 & .37 & 7,434 & 4,332 & 3,101 & .58 & 8,379 & 5,144 & 3,235 & .61 \\
\hline 1987 & 4,494 & 2,298 & 2,196 & .51 & 11,850 & 7,997 & 3,853 & .67 & 13,546 & 9,318 & 4,228 & .69 \\
\hline 1988 & 690 & 296 & 395 & .43 & 1,888 & 1,350 & 538 & .71 & 2,184 & 1,506 & 678 & .69 \\
\hline 1989 & 3,289 & 1,787 & 1,502 & .54 & 4,985 & 3,020 & 1,966 & .61 & 5,436 & 3,318 & 2,118 & .61 \\
\hline 1990 & 5,265 & 3,103 & 2,162 & .59 & 7,951 & 5,426 & 2,525 & .68 & 7,742 & 5,528 & 2,214 & .71 \\
\hline 1991 & 2,503 & 1,182 & 1,321 & .47 & 7,088 & 4,052 & 3,035 & .57 & 7,914 & 4,680 & 3,233 & .59 \\
\hline 1992 & 11,319 & 6,599 & 4,720 & .58 & 26,170 & 18,484 & 7,685 & .71 & 26,619 & 20,153 & 6,466 & .76 \\
\hline 1993 & 2,319 & 1,170 & 1,149 & .50 & 8,731 & 5,249 & 3,482 & .60 & 9,733 & 5,727 & 4,006 & .59 \\
\hline 1994 & 1,646 & 736 & 911 & .45 & 3,399 & 2,233 & 1,166 & .66 & 3,718 & 2,351 & 1,366 & .63 \\
\hline 1995 & 4,474 & 1,881 & 2,594 & .42 & 10,508 & 5,409 & 5,099 & .51 & 11,676 & 6,313 & 5,363 & .54 \\
\hline 1996 & 774 & 346 & 429 & .45 & 1,638 & 1,027 & 611 & .63 & 1,885 & 1,201 & 684 & .64 \\
\hline 1997 & 6,497 & 3,337 & 3,160 & .51 & 13,437 & 9,607 & 3,830 & .72 & 13,656 & 9,880 & 3,776 & .72 \\
\hline 1998 & 2,892 & 951 & 1,941 & .33 & 7,207 & 4,114 & 3,094 & .57 & 7,735 & 4,616 & 3,119 & .60 \\
\hline 1999 & 1,273 & 470 & 804 & .37 & 7,336 & 3,467 & 3,869 & .47 & 8,251 & 3,940 & 4,311 & .48 \\
\hline 2000 & 506 & 150 & 356 & .30 & 1,175 & 640 & 536 & .54 & 1,201 & 667 & 534 & .56 \\
\hline 2001 & 3,253 & 1,428 & 1,825 & .44 & 9,390 & 5,252 & 4,139 & .56 & 9,662 & 5,653 & 4,009 & .58 \\
\hline 2002 & 1,849 & 726 & 1,122 & .39 & 5,249 & 3,004 & 2,244 & .57 & 5,435 & 3,102 & 2,333 & .57 \\
\hline 2003 & 1,336 & 499 & 837 & .37 & 7,758 & 4,123 & 3,634 & .53 & 8,515 & 4,432 & 4,083 & .52 \\
\hline 2004 & 2,605 & 1,000 & 1,605 & .38 & 9,805 & 4,717 & 5,089 & .48 & 10,115 & 4,580 & 5,535 & .45 \\
\hline 2005 & 3,719 & 1,738 & 1,982 & .47 & 10,877 & 7,017 & 3,861 & .65 & 11,280 & 6,944 & 4,336 & .62 \\
\hline
\end{tabular}


in 2000. At the Hempstead station, base flow as a percentage of streamflow ranged from a high of about 72 percent in 1997 to a low of about 47 percent in 1999. At the Richmond station, base flow as a percentage of streamflow ranged from a high of about 76 percent in 1992 to a low of about 48 percent in 1999.

The compositions of streamflow at the Highbank and Hempstead gaging stations were compared graphically using BFI values (tables 3, 4). BFI values for the Brazos River near Highbank were graphed relative to corresponding BFI values for the Brazos River near Hempstead (fig. 4A). This graph shows that for each water year, the BFI value for the Brazos River near Hempstead is greater than the corresponding BFI value for the Brazos River near Highbank, which indicates that a larger percentage of the mean annual flow at the downstream gage (Hempstead) than at the upstream gage (Highbank) is composed of base flow.

The graph of BFI values in figure 4A does not reflect the effect of tributary inflows in the reach between the Highbank and Hempstead stations. To include the effect of tributary inflows, the results of hydrograph separation (annual mean base flow and streamflow) for tributary sites (stations) S1-S6 (fig. 2) were summed and added to those from the Brazos River near Highbank (table 4). The sum of drainage areas for these six stations is 8,731 square miles, which accounts for about 65 percent of the intervening drainage area between the Brazos River near Highbank and the Brazos River near Hempstead stations. The BFI values for the Highbank and tributary stations combined were graphed relative to corresponding BFI values for the Brazos River near Hempstead (fig. 4B). The graph including the effect of tributary inflows is very similar to that without the effect of tributary inflows, again indicating that more of the mean annual flow at the downstream gage (Hempstead) is composed of base flow.

Data to quantify the potential effects on historical streamflows of diversions, return flows, or other anthropogenic activities are not available. To assess whether these factors have affected historical streamflows, BFI values were graphed relative to time. Figure $4 \mathrm{C}$ shows a time series of BFI values for the Brazos River near Hempstead station, and figure 4D shows time series of BFI values for the Brazos River near Highbank station and for the Highbank station plus tributary stations. Visual examination of these time-series graphs does not indicate any discernible trends. To verify the visual result, Mann-Kendall trend tests (Helsel and others, 1992) were run on the time series; no significant trends were indicated (at the .05 level of significance) ( $\mathrm{p}$-value $=.49$ for the Brazos River near Hempstead time series [fig. 4C], p-value $=.70$ for the Brazos River near Highbank time series [fig. 4D], p-value = .83 for the Brazos River near Highbank plus tributary stations time series [fig. 4D]).

The same process was applied to compare the compositions of streamflow at the Brazos River near Hempstead and Brazos River at Richmond gaging stations (fig. 5; table 3). Again, annual BFI values for the two gages were compared graphically (fig. 5A). This graph shows that for each water year, the BFI value for the Brazos River at Richmond is on average about the same as the corresponding BFI value for the Brazos River near Hempstead, which indicates that the percentage of the mean annual flow that is base flow at the downstream gage (Richmond) is not noticeably different from that at the upstream gage (Highbank).

One USGS streamflow-gaging station is on a tributary that enters the Brazos River in the reach between the Hempstead and Richmond stations (site S7, table 1). The drainage area for this station is 376 square miles, or about 31 percent of the intervening drainage area between the Brazos River near Hempstead and Brazos River at Richmond stations. Figure $5 \mathrm{~B}$ shows the graph of BFI values for the Brazos River near Hempstead plus the tributary station relative to the BFI values for the Brazos River at Richmond. The relation in this graph is similar to that of the BFIs without the effect of the tributary (fig. 5A), although it appears slightly biased toward a larger percentage of base flow in streamflow at the Hempstead station than at the Richmond station. Time series of BFI values for the Brazos River at Richmond station (fig. 5C) and for the Brazos River near Hempstead station and the Brazos River near Hempstead station plus the tributary station (fig. 5D) do not show discernible trends, which likely indicates diversions, return flows, or other anthropogenic activities have not measurably affected streamflow in the reach during 1966-2005. Mann-Kendall trend tests run on the time series indicated no significant trends at the .05 level of significance $(\mathrm{p}$-value $=$ .29 for the Brazos River at Richmond time series and p-value $=.77$ for the Brazos River near Hempstead plus one tributary station time series).

Hydrograph separation and graphical evidence from BFI values indicate appreciably more base flow as a percentage of streamflow at the Hempstead station (about 60 percent on average) than at the Highbank station (about 43 percent on average). The reach of the Brazos River between the two stations crosses the outcrops of the Carrizo-Wilcox, Queen City, Sparta, and Yegua-Jackson aquifers, the probable source of the additional base flow. This result is consistent with indications of a gaining reach on potentiometric-surface maps of the Carrizo-Wilcox and Queen City-Sparta aquifers of Garza and others (1987) and with the Texas Board of Water Engineers (1960) qualitative description of the river gaining base flow in the reach, as noted previously in the "Description of Study Area" section. Hydrograph separation and graphical evidence from BFI values also indicate no increase in base flow as a percentage of streamflow in the reach of the Brazos River crossing the Gulf Coast aquifer (Chicot, Evangeline, and Jasper aquifers) compared to that in the adjacent upstream reach. Base flow as a percentage of streamflow at the Richmond station is essentially the same as that at the Hempstead station (about 60 percent on average).

\section{Streamflow Gain and Loss}

Daily mean streamflow of the Brazos River at three stations during the March and August 2006 surveys (08096500 

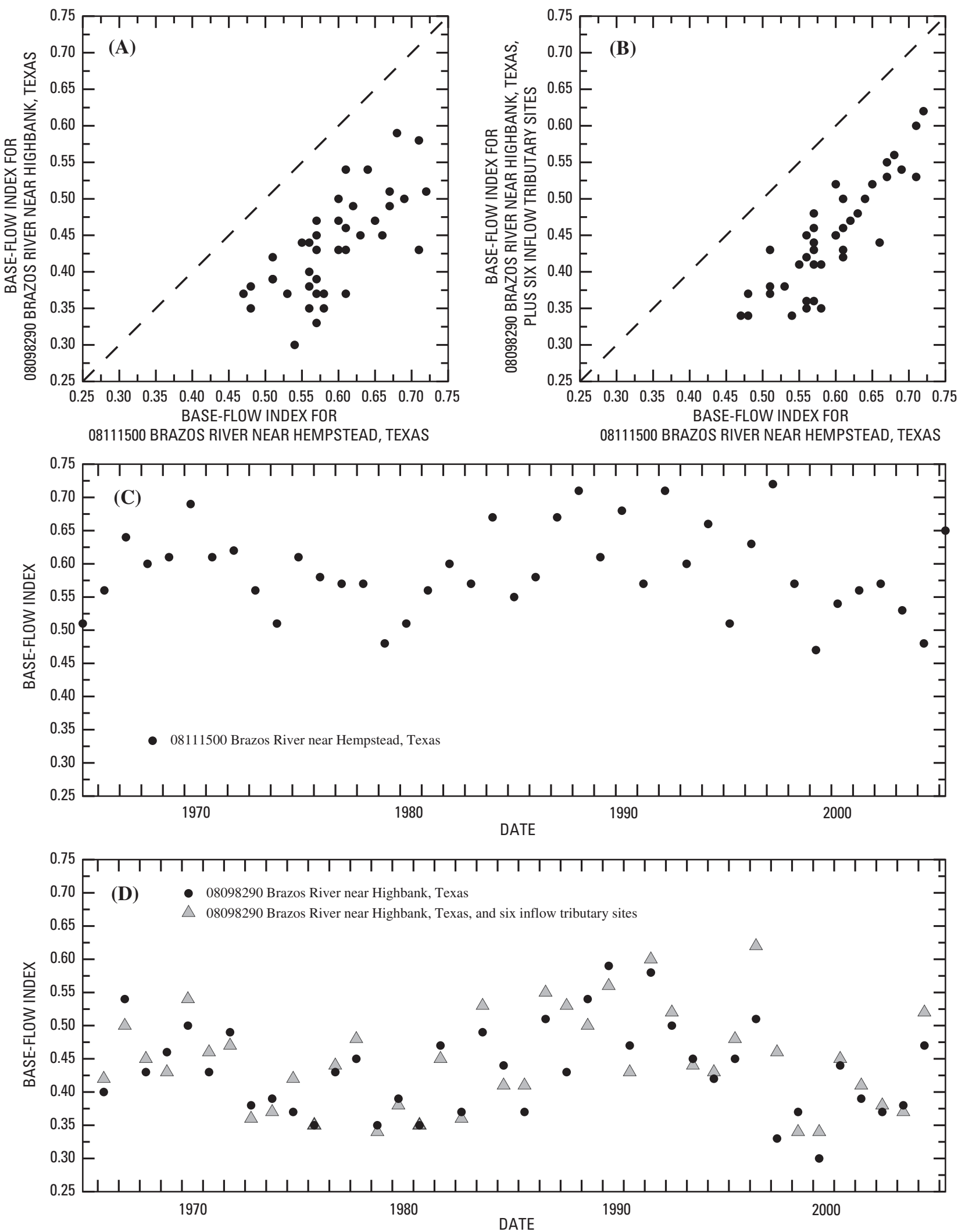

Figure 4. (A) Relation between annual base-flow index for 08098290 Brazos River near Highbank, Texas, and for 08111500 Brazos River near Hempstead, Texas; (B) relation between annual base-flow index for 08098290 Brazos River near Highbank, Texas, plus six inflow tributary sites, and for 08111500 Brazos River near Hempstead, Texas; (C) time series of annual base-flow index for 08111500 Brazos River near Hempstead, Texas, 1966-2005; and (D) time series of annual base-flow index for 08098290 Brazos River near Highbank, Texas, 1966-2005, and for 08098290 Brazos River near Highbank, Texas, plus six inflow tributary sites, 1966-2005. 

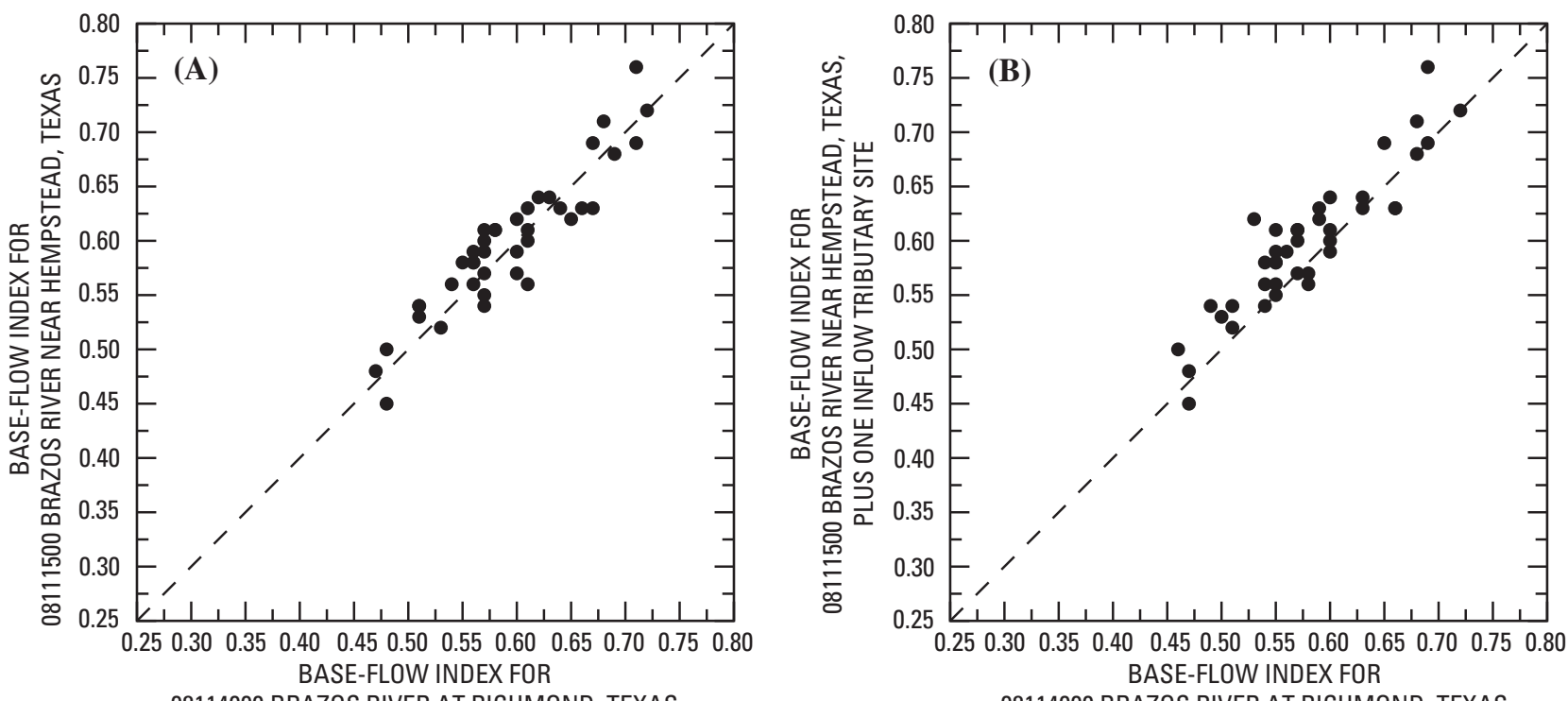

08114000 BRAZOS RIVER AT RICHMOND, TEXAS

08114000 BRAZOS RIVER AT RICHMOND, TEXAS
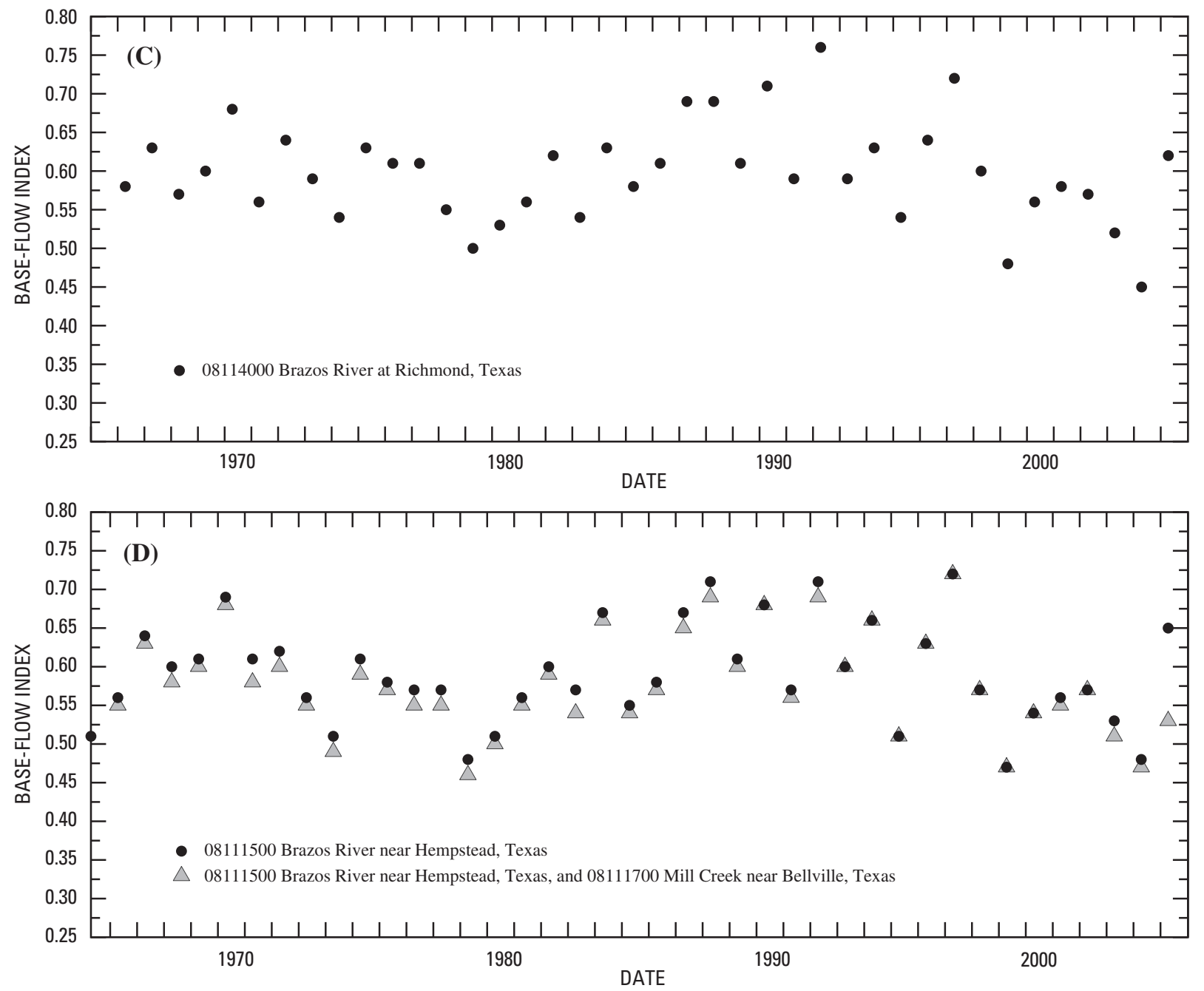

Figure 5. (A) Relation between annual base-flow index for 08111500 Brazos River near Hempstead, Texas, and for 08114000 Brazos River at Richmond, Texas; (B) relation between annual base-flow index for 08111500 Brazos River near Hempstead, Texas, plus one inflow tributary site, and for 08114000 Brazos River at Richmond, Texas; (C) time series of annual base-flow index for 08114000 Brazos River at Richmond, Texas, 1966-2005; and (D) time series of annual base-flow index for 08111500 Brazos River near Hempstead, Texas, 1966-2005, and for 08111500 Brazos River near Hempstead, Texas, plus one inflow tributary site, 1966-2005. 
Table 4. Results of hydrograph separation for 08098290 Brazos River near Highbank, Texas, plus six inflow tributary sites, 1966-2005; and 08111500 Brazos River near Hempstead, Texas, plus one inflow tributary site, 1966-2005.

[Q, annual mean streamflow; $\mathrm{ft}^{3} / \mathrm{s}$, cubic feet per second; BF, base-flow component; RO, runoff component; BFI, base-flow index (defined as BF/Q)]

\begin{tabular}{|c|c|c|c|c|c|c|c|c|}
\hline \multirow{2}{*}{$\begin{array}{l}\text { Water } \\
\text { year }\end{array}$} & \multicolumn{4}{|c|}{$\begin{array}{l}\quad 08098290 \text { Brazos River near } \\
\text { Highbank, Tex., plus six inflow tributary sites' }\end{array}$} & \multicolumn{4}{|c|}{$\begin{array}{c}08111500 \text { Brazos River near Hempstead, Tex., plus one } \\
\text { inflow tributary site }{ }^{2}\end{array}$} \\
\hline & $\begin{array}{c}0 \\
\left(f^{3} / \mathbf{s}\right)\end{array}$ & $\begin{array}{c}\mathrm{BF} \\
(\mathbf{f t} / \mathbf{s})\end{array}$ & $\begin{array}{c}\mathrm{RO} \\
\left(\mathrm{ft}^{3} / \mathrm{s}\right)\end{array}$ & BFI & $\begin{array}{c}0 \\
\left(f^{3} / \mathbf{s}\right)\end{array}$ & $\begin{array}{c}\mathrm{BF} \\
\left(\mathbf{f t} \mathrm{t}^{3} / \mathbf{s}\right)\end{array}$ & $\begin{array}{c}\mathrm{RO} \\
\left(\mathrm{ft}^{3} / \mathrm{s}\right)\end{array}$ & BFI \\
\hline 1966 & 6,822 & 2,852 & 3,970 & 0.42 & 8,334 & 4,643 & 3,690 & 0.56 \\
\hline 1967 & 1,538 & 776 & 762 & .50 & 1,844 & 1,170 & 674 & .63 \\
\hline 1968 & 9,581 & 4,300 & 5,281 & .45 & 12,937 & 7,567 & 5,370 & .58 \\
\hline 1969 & 5,158 & 2,199 & 2,959 & .43 & 7,812 & 4,677 & 3,135 & .60 \\
\hline 1970 & 4,999 & 2,716 & 2,283 & .54 & 6,425 & 4,347 & 2,077 & .68 \\
\hline 1971 & 1,013 & 470 & 543 & .46 & 1,545 & 910 & 635 & .59 \\
\hline 1972 & 3,176 & 1,506 & 1,671 & . 47 & 3,889 & 2,340 & 1,548 & .60 \\
\hline 1973 & 5,778 & 2,100 & 3,679 & .36 & 8,591 & 4,680 & 3,911 & .54 \\
\hline 1974 & 3,966 & 1,473 & 2,493 & .37 & 7,182 & 3,522 & 3,660 & .49 \\
\hline 1975 & 9,858 & 4,127 & 5,731 & .42 & 12,902 & 7,666 & 5,236 & .59 \\
\hline 1976 & 4,099 & 1,436 & 2,662 & .35 & 5,580 & 3,179 & 2,400 & .57 \\
\hline 1977 & 7,184 & 3,174 & 4,010 & .44 & 10,431 & 5,757 & 4,674 & .55 \\
\hline 1978 & 887 & 423 & 463 & .48 & 1,661 & 923 & 738 & .56 \\
\hline 1979 & 5,943 & 2,047 & 3,896 & .34 & 10,351 & 4,776 & 5,575 & .46 \\
\hline 1980 & 2,440 & 923 & 1,517 & .38 & 3,892 & 1,961 & 1,931 & .50 \\
\hline 1981 & 3,464 & 1,222 & 2,242 & .35 & 4,689 & 2,574 & 2,116 & .55 \\
\hline 1982 & 6,376 & 2,881 & 3,496 & .45 & 7,583 & 4,438 & 3,145 & .59 \\
\hline 1983 & 2,417 & 879 & 1,538 & .36 & 4,765 & 2,591 & 2,173 & .54 \\
\hline 1984 & 590 & 314 & 275 & .53 & 1,273 & 839 & 433 & .66 \\
\hline 1985 & 3,654 & 1,495 & 2,160 & .41 & 6,320 & 3,423 & 2,896 & .54 \\
\hline 1986 & 5,535 & 2,282 & 3,253 & .41 & 7,671 & 4,380 & 3,291 & .57 \\
\hline 1987 & 8,886 & 4,856 & 4,030 & .55 & 12,240 & 8,063 & 4,177 & .66 \\
\hline 1988 & 1,297 & 691 & 606 & .53 & 2,003 & 1,379 & 625 & .69 \\
\hline 1989 & 4,295 & 2,142 & 2,154 & .50 & 5,061 & 3,038 & 2,023 & .60 \\
\hline 1990 & 7,122 & 4,018 & 3,104 & .56 & 7,979 & 5,440 & 2,539 & .68 \\
\hline 1991 & 4,495 & 1,937 & 2,557 & .43 & 7,357 & 4,088 & 3,270 & .56 \\
\hline 1992 & 20,987 & 12,523 & 8,464 & .60 & 26,836 & 18,593 & 8,243 & .69 \\
\hline 1993 & 5,174 & 2,700 & 2,475 & .52 & 8,731 & 5,249 & 3,482 & .60 \\
\hline 1994 & 2,636 & 1,169 & 1,467 & .44 & 3,399 & 2,233 & 1,166 & .66 \\
\hline 1995 & 7,552 & 3,216 & 4,335 & .43 & 10,508 & 5,409 & 5,099 & .51 \\
\hline 1996 & 1,237 & 592 & 645 & .48 & 1,638 & 1,027 & 611 & .63 \\
\hline 1997 & 11,872 & 7,344 & 4,527 & .62 & 13,437 & 9,607 & 3,830 & .71 \\
\hline 1998 & 5,701 & 2,614 & 3,087 & .46 & 7,207 & 4,114 & 3,094 & .57 \\
\hline 1999 & 4,067 & 1,389 & 2,678 & .34 & 7,336 & 3,467 & 3,869 & .47 \\
\hline 2000 & 853 & 289 & 564 & .34 & 1,175 & 640 & 536 & .54 \\
\hline 2001 & 6,591 & 2,936 & 3,655 & .45 & 9,571 & 5,294 & 4,277 & .55 \\
\hline 2002 & 3,993 & 1,649 & 2,345 & .41 & 5,342 & 3,032 & 2,310 & .57 \\
\hline 2003 & 3,819 & 1,472 & 2,347 & .39 & 8,216 & 4,191 & 4,024 & .51 \\
\hline 2004 & 5,332 & 1,979 & 3,353 & .37 & 10,081 & 4,784 & 5,297 & .47 \\
\hline 2005 & 8,235 & 4,293 & 3,942 & .52 & 11,065 & 7,065 & 3,999 & .64 \\
\hline
\end{tabular}

${ }^{1}$ Sites S1-S6 (fig. 2, table 1).

${ }^{2}$ Site S7 (fig. 2, table 1). 
A. 08096500 Brazos River at Waco, Texas
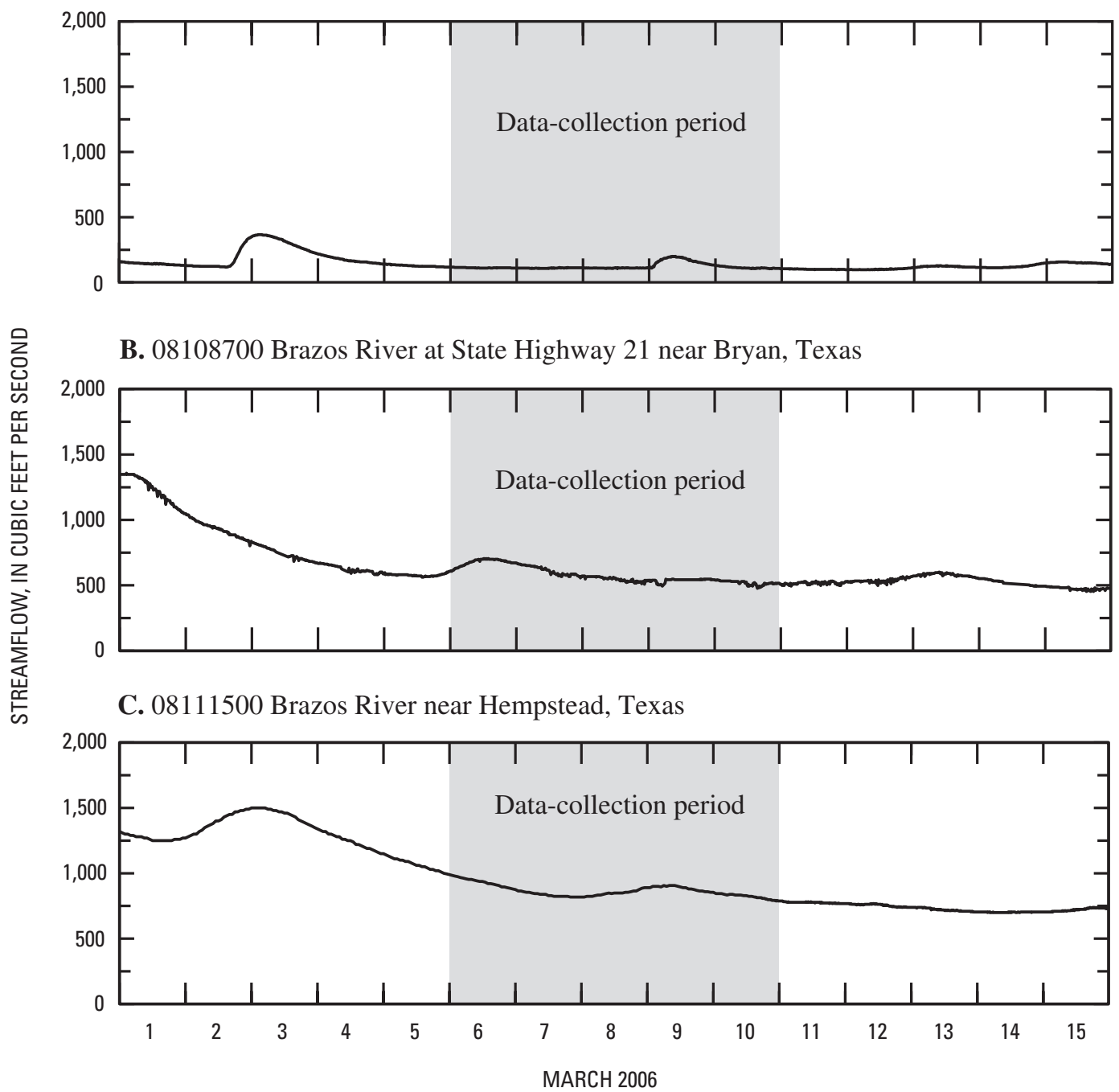

Figure 6. Daily mean streamflow for March 1-15, 2006, at (A) 08096500 Brazos River at Waco, Texas; (B) 08108700 Brazos River at State Highway 21 near Bryan, Texas; and (C) 08111500 Brazos River near Hempstead, Texas.

Brazos River at Waco, 08108700 Brazos River at State Highway 21 near Bryan, and 08111500 Brazos River near Hempstead) (figs. 6, 7) was relatively stable, except at the Waco station during the August survey. At the Waco station, streamflow was affected most days during the August survey by releases from upstream reservoirs for hydroelectric power generation.

Measurements of streamflow at the 36 sites along the main stem of the Brazos River from the March survey (table 5) are graphed relative to downstream distance in figure 8 . Streamflow increases from upstream to downstream except at the downstream end of the study reach. An appreciable increase in Brazos River flow (173 cubic feet per second) occurred between sites B12 and B13 (table 5), the reach bracketing the confluence with Little River, the largest tributary. Another large increase in flow (258 cubic feet per second) occurred between sites B15 and B16 (river miles 269-258, fig. 8) that is not a result of tributary inflow.
Measurements of streamflow at 28 of the 36 sites along the main stem of the Brazos River from the August survey (table 6) are graphed relative to downstream distance in figure 9. The graph shows the same general pattern of increasing streamflow from upstream to downstream, except at the downstream end of the study reach, as in the March survey, although the minimum flows at the upstream end are larger and the maximum flows toward the downstream end are lower. Larger minimum flows at the upstream end of the study reach likely are the result of releases from upstream reservoirs (fig. 7); and lower maximum flows likely are the result of generally drier conditions in August. Little River inflow was substantially less than in the March survey, and the increase in flow between sites B15 and B16 (table 6) also was substantially less than in the March survey.

Streamflow gains and losses for reaches defined by pairs of sites on the Brazos River were computed for 35 reaches during the March survey (table 7) and for 27 reaches 
A. 08096500 Brazos River at Waco, Texas

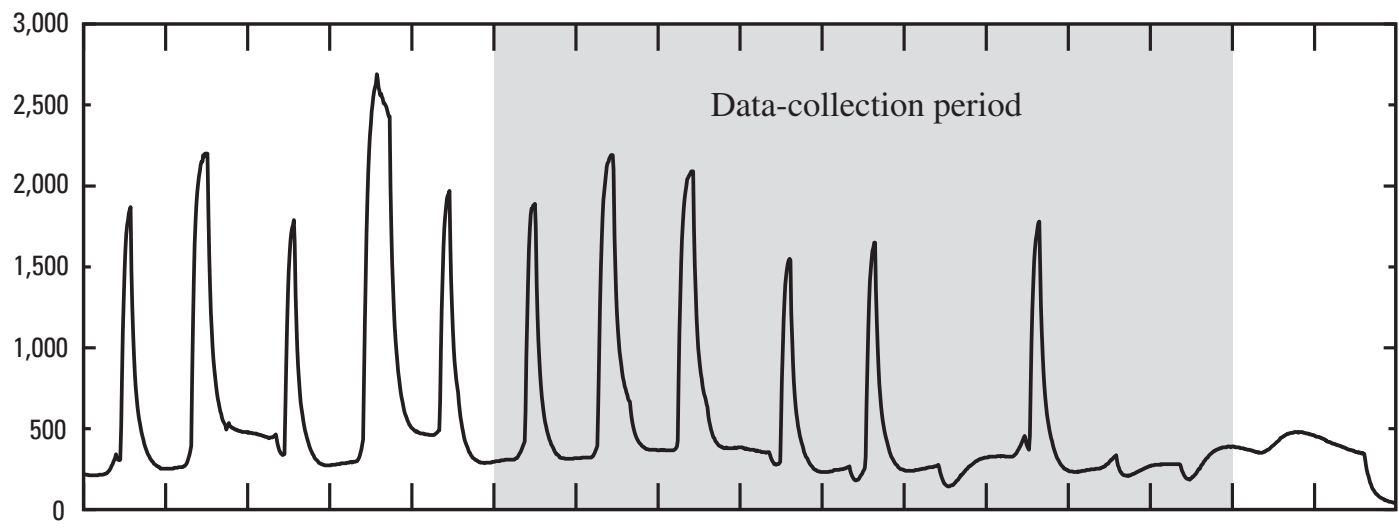

B. 08108700 Brazos River at State Highway 21 near Bryan, Texas

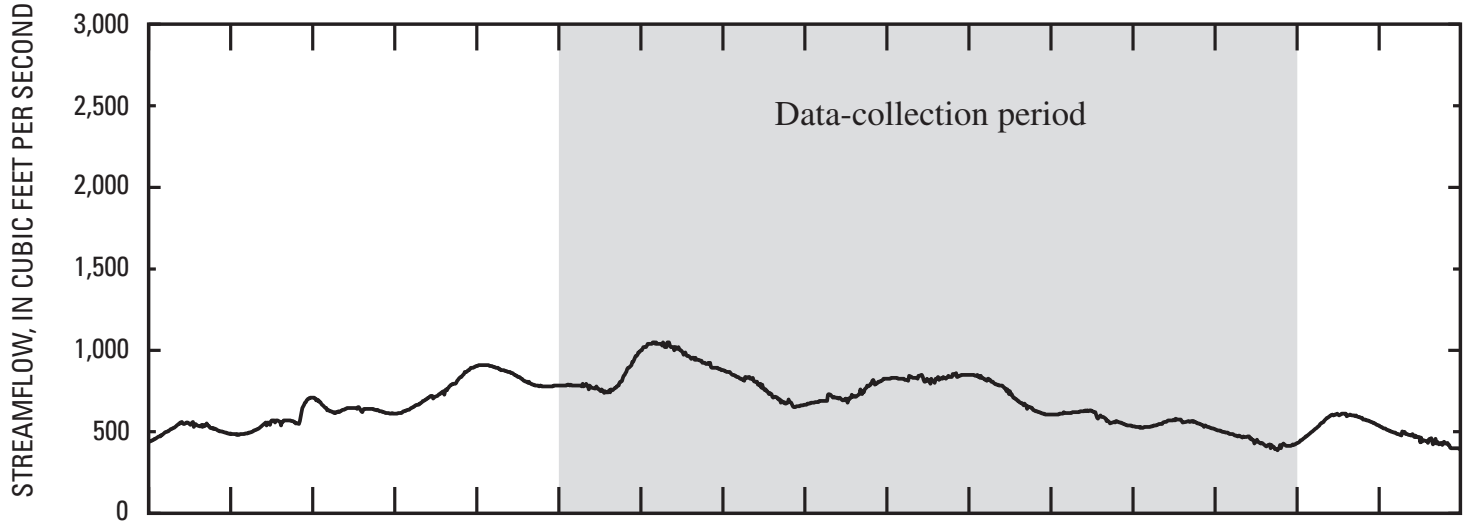

C. 08111500 Brazos River near Hempstead, Texas

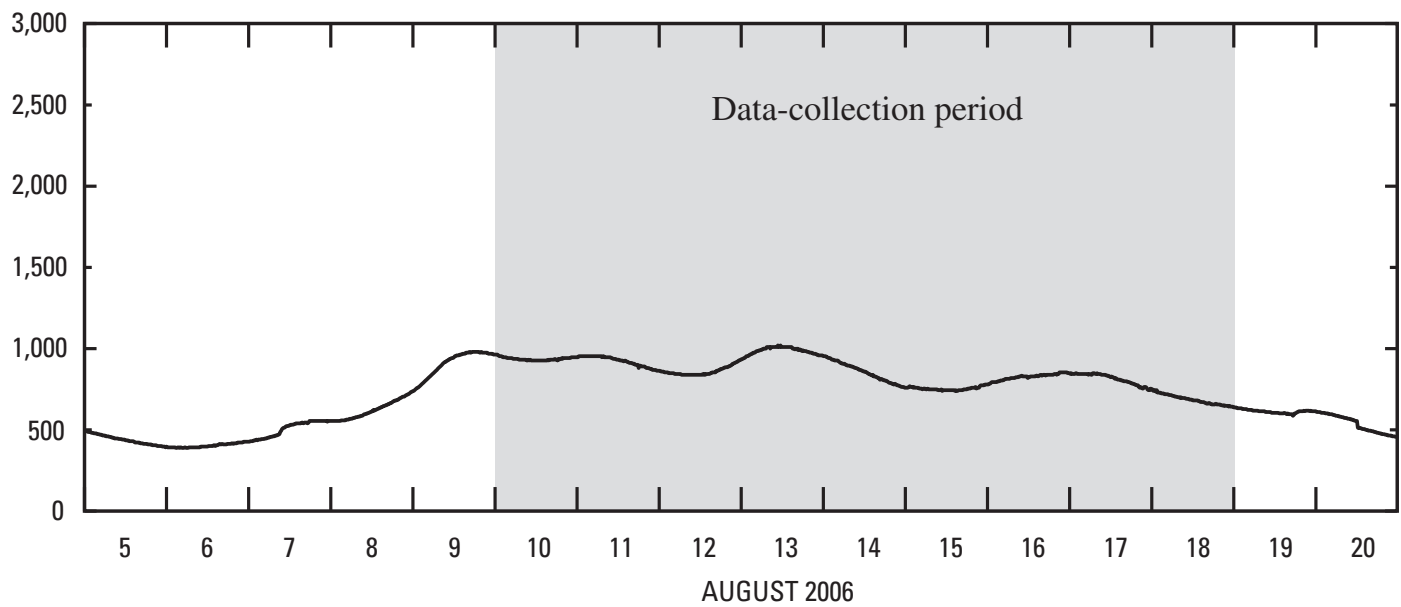

Figure 7. Daily mean streamflow for August 5-20, 2006, at (A) 08096500 Brazos River at Waco, Texas; (B) 08108700 Brazos River at State Highway 21 near Bryan, Texas; and (C) 08111500 Brazos River near Hempstead, Texas.

during the August survey (table 8). Also listed is the potential error for each pair of measurements used to compute a gain or loss. Only gains or losses in reaches for which the difference between upstream and downstream measurements exceeds the potential measurement error are considered verified. Based on this criterion, five of 35 stream reaches were verifiably gaining streamflow and none were verifiably losing streamflow during the March survey (fig. 10). Four of the five gaining reaches are in the outcrop areas of the Carrizo-Wilcox and Yegua-Jackson aquifers. The reach gaining the most flow (B15-B16, 258 cubic feet per second) is in the Yegua-Jackson aquifer outcrop. No reason is known for the gaining reach at Waco, which is not in an aquifer outcrop. 


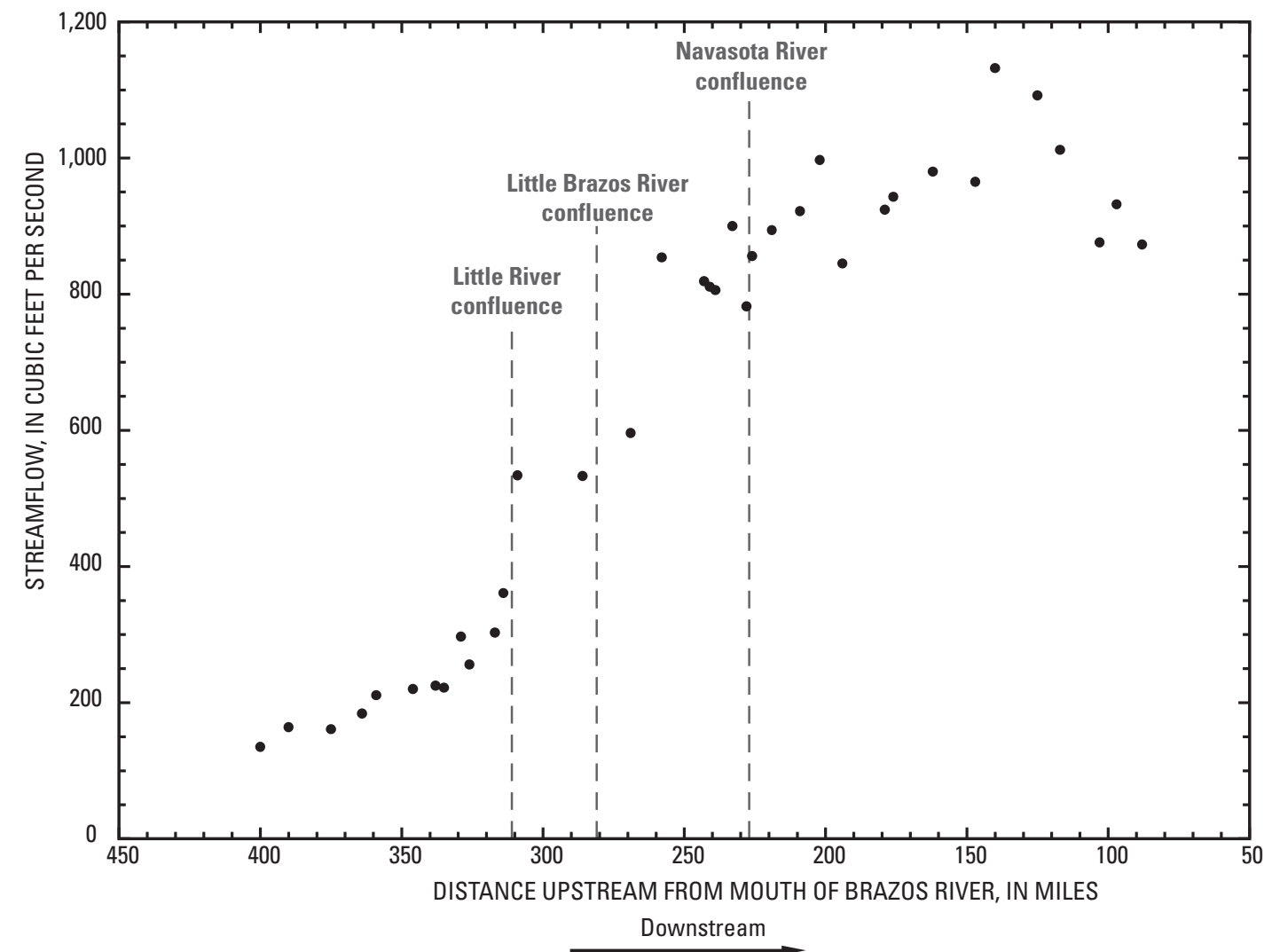

Figure 8. Instantaneous streamflow at 36 sites on the Brazos River, McLennan County to Fort Bend County, Texas, March 6-10, 2006.

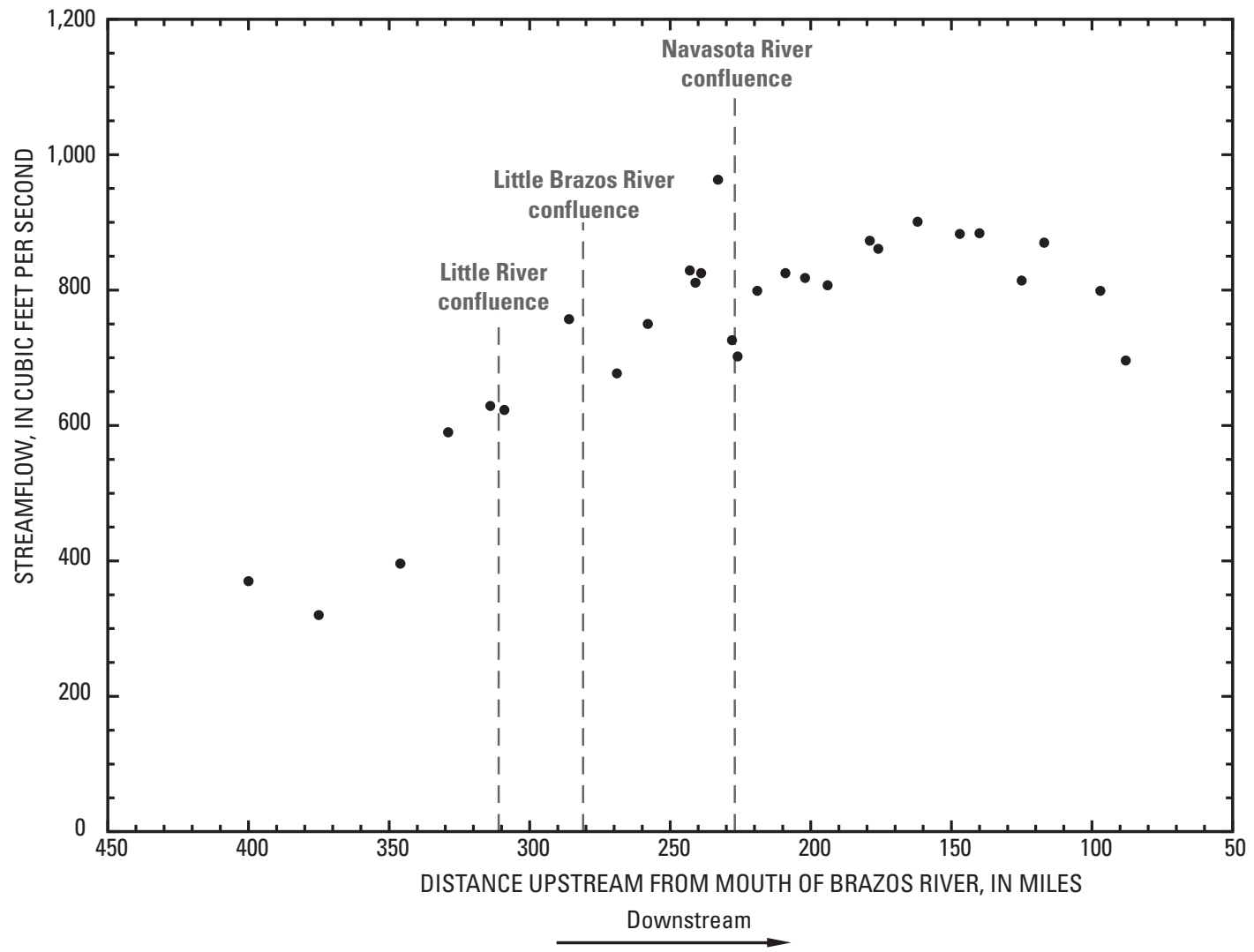

Figure 9. Instantaneous streamflow at 28 sites on the Brazos River, McLennan County to Fort Bend County, Texas, August 10-18, 2006. 


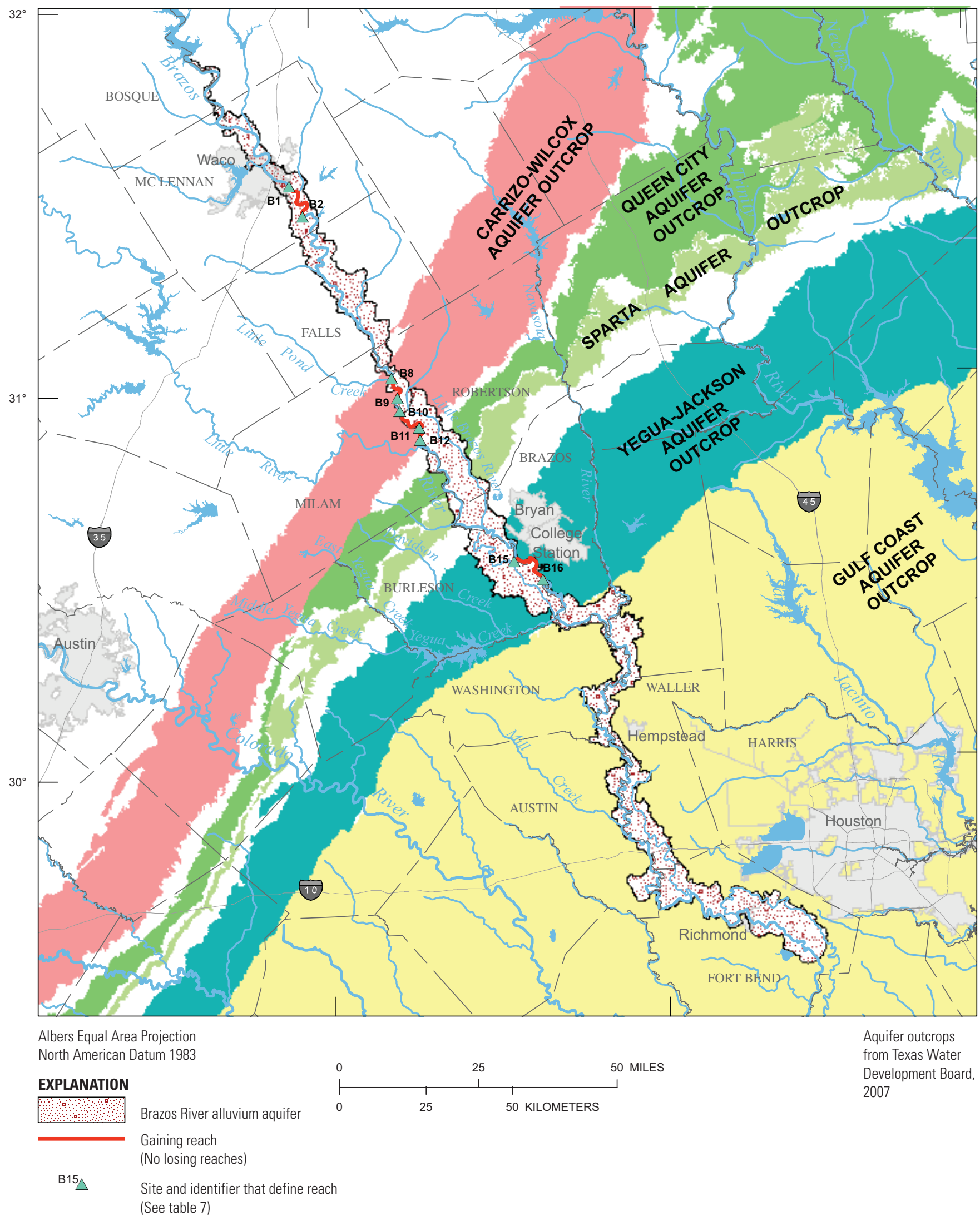

Figure 10. Reaches of the Brazos River, McLennan County to Fort Bend County, Texas, identified as gaining or losing streamflow, March 6-10, 2006. 


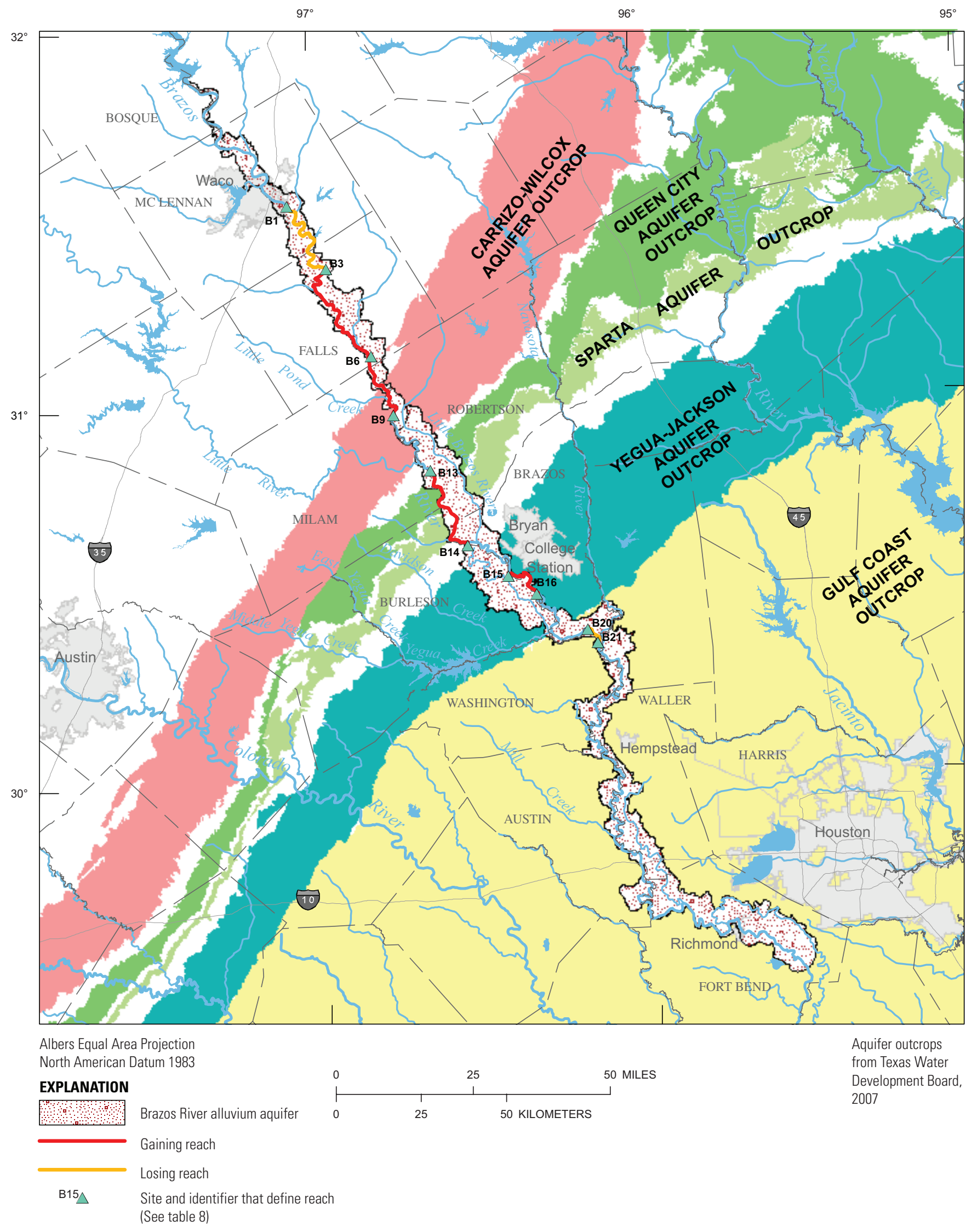

Figure 11. Reaches of the Brazos River, McLennan County to Fort Bend County, Texas, identified as gaining or losing streamflow, August 10-18, 2006. 
For the August survey, four stream reaches were verifiably gaining streamflow and two were losing streamflow (fig. 11). Because some measurement sites in the March survey were not used in the August survey, the number of reaches defined by site pairs is 27, and thus some reaches are longer than those of the March survey. Although the August survey reaches are not everywhere comparable to the March survey reaches, the gaining reaches of the August survey generally match those of the March survey-occurring mostly in the outcrops of the aquifers. The reach gaining the most flow (B6-B9, 194 cubic feet per second) (fig. 11) begins upstream from the outcrop of the Carrizo-Wilcox aquifer and contains the relatively short gaining reach defined by sites B8 and B9 (fig. 10) in the outcrop of the Carrizo-Wilcox aquifer from the March survey. The reach gaining the second-most flow (B13-B14, 134 cubic feet per second) is in the outcrops of the Queen City and Sparta aquifers; that reach was not a gaining reach in the March survey. The reach defined by sites B15 and B16 in the Yegua-Jackson aquifer outcrop, which had the largest gain during the March survey, gained 73 cubic feet per second during the August survey. No reasons are known for the streamflow losses that occurred in the reach defined by sites B1 and B3 at the upstream end of the study reach and in the reach defined by sites B20 and B21 in the outcrop of the Gulf Coast aquifer.

The results of the synoptic gain and loss surveys are consistent with the results of the base-flow analysis (hydrograph separation) of historical streamflow and with the findings of Garza and others (1987) and the Texas Board of Water Engineers (1960). Appreciable increases in streamflow, apparently the result of increases in base flow, occur in the reach of the Brazos River that crosses the outcrops of the Carrizo-Wilcox, Queen City, Sparta, and Yegua-Jackson aquifers. The gain and loss surveys also yielded no evidence for appreciable streamflow gains or losses in the outcrop of the Gulf Coast aquifer, except for the relatively large, unexplained loss (237 cubic feet per second) in the reach defined by sites B20 and B21 in the August survey.

Table 5. Instantaneous streamflow, water temperature, and specific conductance for sites on the Brazos River and selected tributaries, McLennan County to Fort Bend County, Texas, March 6-10, 2006.

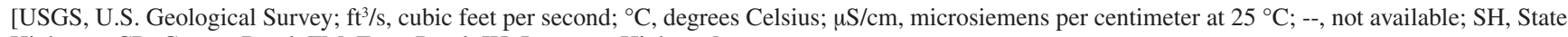
Highway; CR, County Road; FM, Farm Road; IH, Interstate Highway]

\begin{tabular}{|c|c|c|c|c|c|}
\hline $\begin{array}{c}\text { Site } \\
\text { identifier } \\
\text { (fig. 2) }\end{array}$ & USGS station name & Date & $\begin{array}{c}\text { Streamflow, } \\
\text { instantaneous } \\
\left(\mathrm{ft}^{3} / \mathbf{s}\right)\end{array}$ & $\begin{array}{c}\text { Water } \\
\text { temperature } \\
\left({ }^{\circ} \mathrm{C}\right)\end{array}$ & $\begin{array}{c}\text { Specific } \\
\text { conductance } \\
(\mu \mathrm{S} / \mathrm{cm})\end{array}$ \\
\hline $\mathrm{T} 1$ & Tehuacana Creek at SH 6 near Waco, Tex. & March 6, 2006 & .107 & 18.0 & 755 \\
\hline $\mathrm{T} 2$ & Flat Creek at CR 3400 near Robinson, Tex. & March 6, 2006 & .802 & 19.3 & 384 \\
\hline $\mathrm{T} 3$ & Monas Creek at FM 1860 near Riesel, Tex. & March 7, 2006 & .168 & 17.2 & 727 \\
\hline $\mathrm{T} 4$ & Castleman Creek near Robinson, Tex. & March 7, 2006 & .439 & 23.8 & 704 \\
\hline $\mathrm{T} 5$ & Bull Hide Creek at FM 434 near Golinda, Tex. & March 7, 2006 & 1.00 & 18.7 & 925 \\
\hline B3 & Brazos River near Golinda, Tex. & March 7, 2006 & 159 & 21.7 & 2,240 \\
\hline B5 & Brazos River at FM 712 near Marlin, Tex. & March 9, 2006 & 209 & 23.0 & 1,850 \\
\hline $\mathrm{T} 8$ & Mussel Run Creek near Highbank, Tex. & March 7, 2006 & 1.59 & 20.4 & 1,400 \\
\hline B6 & Brazos River near Highbank, Tex. & March 9, 2006 & 218 & 21.3 & 1,860 \\
\hline B7 & Brazos River below FM 1373 near Baileyville, Tex. & March 9, 2006 & 223 & 19.4 & 2,090 \\
\hline B8 & Brazos River above Pond Creek near Baileyville, Tex. & March 9, 2006 & 220 & 20.8 & 2,140 \\
\hline T9 & Pond Creek at FM 2027 near Baileyville, Tex. & March 9, 2006 & .180 & 21.1 & 2,470 \\
\hline B9 & Brazos River at FM 979 near Calvert, Tex. & March 6, 2006 & 295 & 18.9 & 2,470 \\
\hline
\end{tabular}


Table 5. Instantaneous streamflow, water temperature, and specific conductance for sites on the Brazos River and selected tributaries, McLennan County to Fort Bend County, Texas, March 6-10, 2006-Continued.

\begin{tabular}{|c|c|c|c|c|c|}
\hline $\begin{array}{c}\text { Site } \\
\text { identifier } \\
\text { (fig. 2) }\end{array}$ & USGS station name & Date & $\begin{array}{c}\text { Streamflow, } \\
\text { instantaneous } \\
\left(\mathrm{ft}^{3} / \mathbf{s}\right)\end{array}$ & $\begin{array}{c}\text { Water } \\
\text { temperature } \\
\left({ }^{\circ} \mathrm{C}\right)\end{array}$ & $\begin{array}{c}\text { Specific } \\
\text { conductance } \\
(\mu \mathrm{S} / \mathrm{cm})\end{array}$ \\
\hline B12 & Brazos River at FM 485 near Hearne, Tex. & March 6, 2006 & 359 & 21.1 & 2,240 \\
\hline $\mathrm{T} 10$ & Little River at CR 263 near Gause, Tex. & March 6, 2006 & 239 & 22.2 & 659 \\
\hline B13 & Brazos River at Valley Junction, Tex. & March 7, 2006 & 532 & 20.6 & 1,660 \\
\hline B14 & Brazos River at SH 21 near Bryan, Tex. & March 10, 2006 & 531 & 20.0 & 1,630 \\
\hline T11 & Little Brazos River at SH 21 near Bryan, Tex. & March 10, 2006 & 35.6 & 20.3 & 794 \\
\hline B15 & Brazos River near College Station, Tex. & March 10, 2006 & 594 & 20.6 & 1,550 \\
\hline B16 & Brazos River at Batts Ferry near Wellborn, Tex. & March 7, 2006 & 852 & 21.0 & 1,360 \\
\hline B17 & Brazos River near FM 159 near Clay, Tex. & March 7, 2006 & 817 & 23.0 & 1,510 \\
\hline $\mathrm{T} 12$ & Yegua Creek at FM 50 near Clay, Tex. & March 7, 2006 & 5.26 & 23.0 & 747 \\
\hline B 18 & Brazos River near FM 1955 near Clay, Tex. & March 7, 2006 & 809 & 24.2 & 1,500 \\
\hline B19 & Brazos River at Rogers Plantation near Millican, Tex. & March 7, 2006 & 804 & 22.0 & 1,700 \\
\hline B20 & Brazos River near FM 159 near Millican, Tex. & March 7, 2006 & 898 & 21.7 & 1,930 \\
\hline B21 & Brazos River at SH 105 near Washington, Tex. & March 8, 2006 & 780 & 20.3 & 1,950 \\
\hline $\mathrm{T} 13$ & Navasota River below SH 105 near Washington, Tex. & March 8, 2006 & 93.0 & 20.5 & 566 \\
\hline B 22 & Brazos River below Navasota River near Washington, Tex. & March 8, 2006 & 854 & 20.4 & 1,810 \\
\hline B23 & Brazos River at Old River Road near Courtney, Tex. & March 8, 2006 & 892 & 21.7 & -- \\
\hline B24 & Brazos River near FM 2726 near Courtney, Tex. & March 8, 2006 & 920 & 21.8 & 1,790 \\
\hline B25 & Brazos River near FM 1736 near Hempstead, Tex. & March 8, 2006 & 995 & 22.7 & 1,740 \\
\hline B26 & Brazos River near Hempstead, Tex. & March 9, 2006 & 843 & 21.4 & 1,740 \\
\hline $\mathrm{T} 14$ & Caney Creek at FM 1371 near Hempstead, Tex. & March 8, 2006 & 1.80 & 23.0 & 589 \\
\hline B27 & Brazos River at SH 159 near Hempstead, Tex. & March 9, 2006 & 922 & 20.8 & 1,730 \\
\hline B28 & Brazos River near FM 1887 near Hempstead, Tex. & March 9, 2006 & 941 & 23.5 & 1,510 \\
\hline $\mathrm{T} 15$ & Clear Creek near FM 1887 near Hempstead, Tex. & March 9, 2006 & 8.84 & 21.5 & 456 \\
\hline T16 & Piney Creek at FM 331 near Burleigh, Tex. & March 8, 2006 & 3.58 & 26.5 & 199 \\
\hline B29 & Brazos River at FM 529 near Burleigh, Tex. & March 9, 2006 & 978 & 22.5 & 1,750 \\
\hline $\mathrm{T} 17$ & Mill Creek at FM 331 near Burleigh, Tex. & March 9, 2006 & 20.8 & 22.0 & 513 \\
\hline B30 & Brazos River at FM 1458 at San Felipe, Tex. & March 8, 2006 & 963 & 21.4 & 1,860 \\
\hline B31 & Brazos River at IH-10 near Brookshire, Tex. & March 8, 2006 & 1,130 & 22.4 & 1,830 \\
\hline B32 & Brazos River at FM 1093 at Simonton, Tex. & March 8, 2006 & 1,090 & 23.3 & 1,790 \\
\hline B33 & Brazos River at FM 1489 near Simonton, Tex. & March 9, 2006 & 1,010 & 22.6 & 1,820 \\
\hline $\mathrm{T} 18$ & Bessies Creek at FM 1093 near Fulshear, Tex. & March 8, 2006 & 2.48 & 24.2 & 320 \\
\hline T19 & Jones Creek at Bois du Arc Road near Fulshear, Tex. & March 9, 2006 & 1.00 & -- & -- \\
\hline B34 & Brazos River at CR near FM 359 near Rosenberg, Tex. & March 9, 2006 & 874 & 22.9 & 1,790 \\
\hline B35 & Brazos River at FM 723 near Rosenberg, Tex. & March 9, 2006 & 930 & 24.1 & 1,730 \\
\hline B36 & Brazos River at Richmond, Tex. & March 9, 2006 & 871 & 24.2 & 1,520 \\
\hline
\end{tabular}


Table 6. Instantaneous streamflow for sites on the Brazos River and selected tributaries, McLennan County to Fort Bend County, Texas, August 10-18, 2006.

[Negative discharge indicates irrigation pumpage from the Brazos River. USGS, U.S. Geological Survey; $\mathrm{ft}^{3} / \mathrm{s}$, cubic feet per second; SH, State Highway; CR, County Road; --, not available; FM, Farm Road; IH, Interstate Highway]

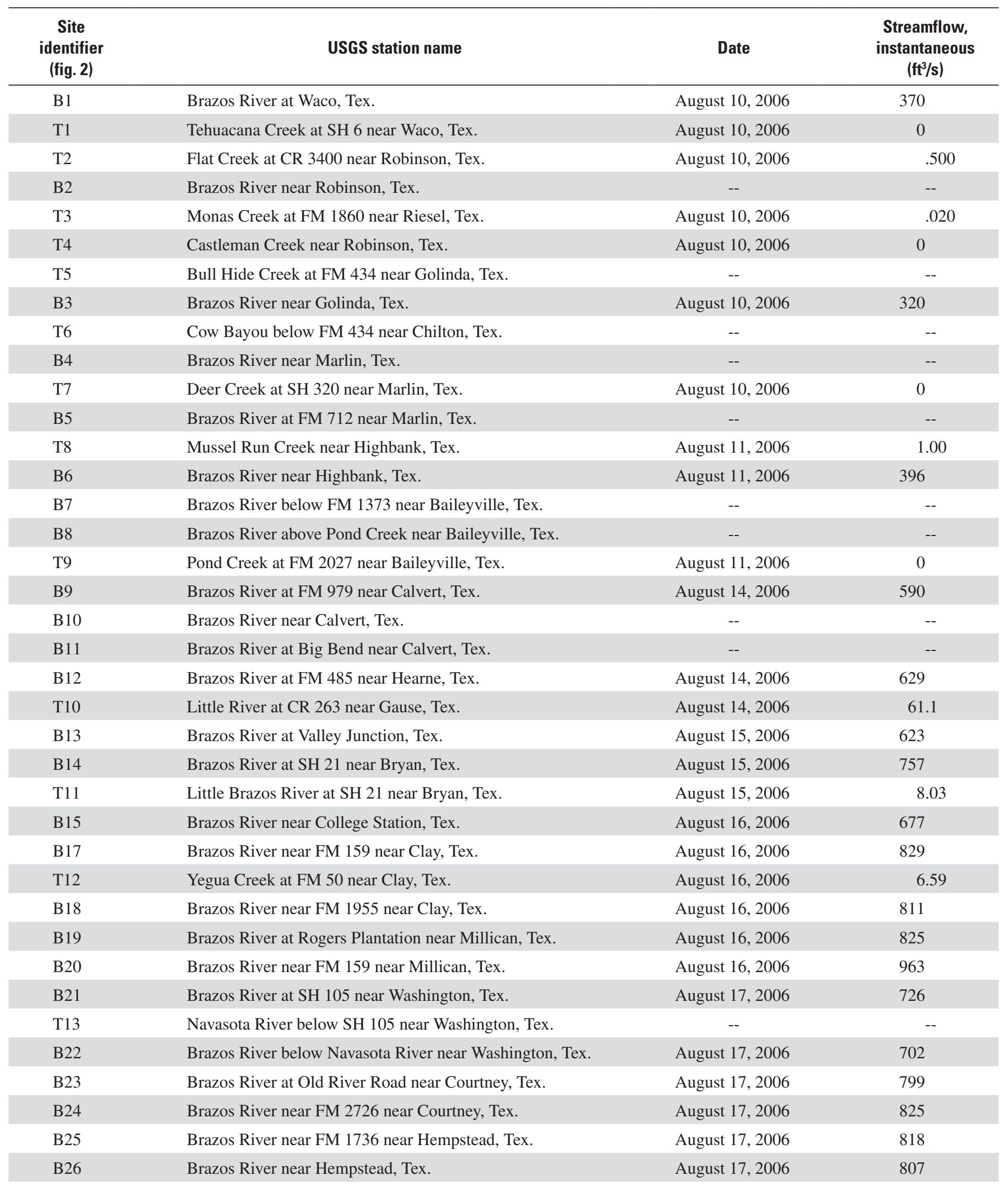


Table 6. Instantaneous streamflow for sites on the Brazos River and selected tributaries, McLennan County to Fort Bend County, Texas, August 10-18, 2006-Continued.

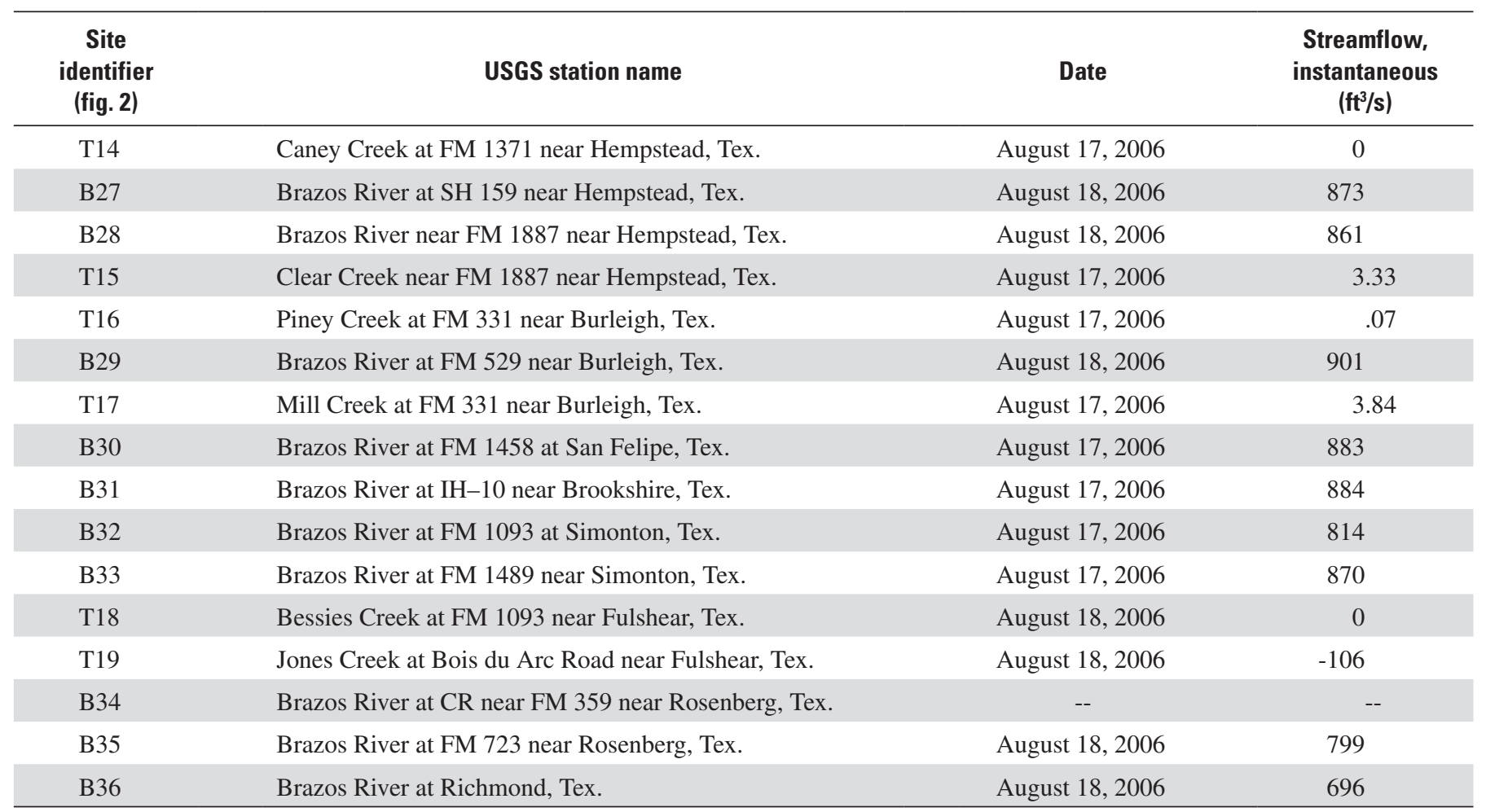

Table 7. Streamflow gains and losses computed for 35 reaches on the Brazos River, McLennan County to Fort Bend County, Texas, March 6-10, 2006.

[Bold font indicates gain or loss that is greater than potential measurement error for that particular reach. mi, miles; $\mathrm{ft}^{3} / \mathrm{s}$, cubic feet per second; --, not applicable]

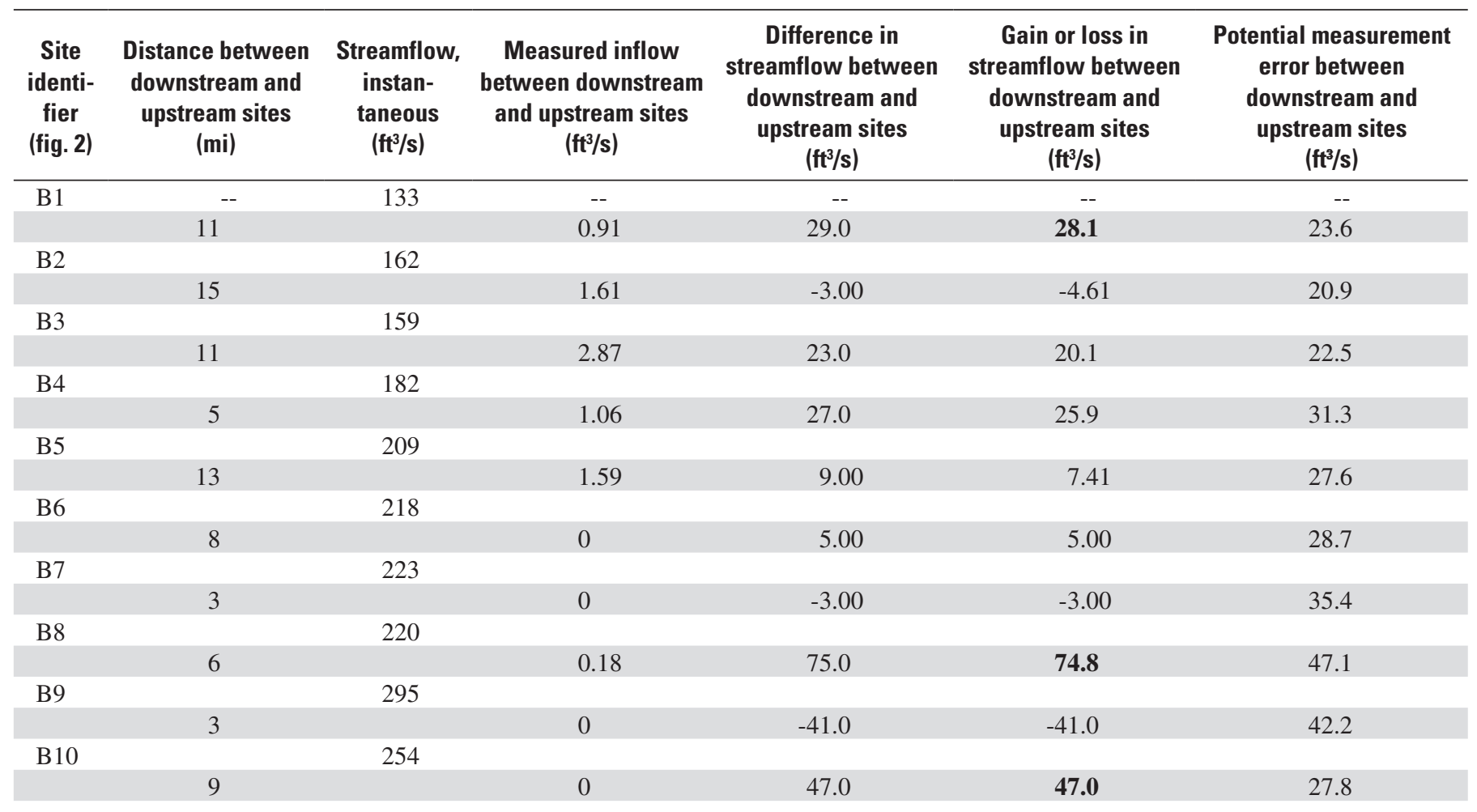


Table 7. Streamflow gains and losses computed for 35 reaches on the Brazos River, McLennan County to Fort Bend County, Texas, March 6-10, 2006-Continued.

\begin{tabular}{|c|c|c|c|c|c|c|}
\hline $\begin{array}{l}\text { Site } \\
\text { identi- } \\
\text { fier } \\
\text { (fig. 2) }\end{array}$ & $\begin{array}{c}\text { Distance between } \\
\text { downstream and } \\
\text { upstream sites } \\
\text { (mi) }\end{array}$ & $\begin{array}{l}\text { Streamflow, } \\
\text { instan- } \\
\text { taneous } \\
\left(\mathrm{ft}^{3} / \mathrm{s}\right)\end{array}$ & $\begin{array}{c}\text { Measured inflow } \\
\text { between downstream } \\
\text { and upstream sites } \\
\left(\mathrm{ft}^{3} / \mathrm{s}\right)\end{array}$ & $\begin{array}{c}\text { Difference in } \\
\text { streamflow between } \\
\text { downstream and } \\
\text { upstream sites } \\
\left(\mathrm{ft}^{3} / \mathrm{s}\right)\end{array}$ & $\begin{array}{c}\text { Gain or loss in } \\
\text { streamflow between } \\
\text { downstream and } \\
\text { upstream sites } \\
\left(\mathrm{ft}^{3} / \mathrm{s}\right)\end{array}$ & $\begin{array}{l}\text { Potential measurement } \\
\text { error between } \\
\text { downstream and } \\
\text { upstream sites } \\
\left(\mathrm{ft}^{3} / \mathrm{s}\right)\end{array}$ \\
\hline \multirow[t]{2}{*}{ B11 } & & 301 & & & & \\
\hline & 3 & & 0 & 58.0 & 58.0 & 43.8 \\
\hline \multirow[t]{2}{*}{ B12 } & & 359 & & & & \\
\hline & 5 & & 239 & 173 & -66.0 & 71.3 \\
\hline \multirow[t]{2}{*}{ B13 } & & 532 & & & & \\
\hline & 23 & & 0 & -1.00 & -1.00 & 85.0 \\
\hline \multirow[t]{2}{*}{ B14 } & & 531 & & & & \\
\hline & 17 & & 35.6 & 63.0 & 27.4 & 90.0 \\
\hline \multirow[t]{2}{*}{ B15 } & & 594 & & & & \\
\hline & 11 & & 0 & 258 & 258 & 116 \\
\hline \multirow[t]{2}{*}{ B16 } & & 852 & & & & \\
\hline & 15 & & 0 & -35.0 & -35.0 & 134 \\
\hline \multirow[t]{2}{*}{ B17 } & & 817 & & & & \\
\hline & 2 & & 5.26 & -8.00 & -13.3 & 130 \\
\hline \multirow[t]{2}{*}{ B18 } & & 809 & & & & \\
\hline & 2 & & 0 & -5.00 & -5.00 & 129 \\
\hline \multirow{2}{*}{ B19 } & & 804 & & & & \\
\hline & 6 & & 0 & 94.0 & 94.0 & 136 \\
\hline \multirow[t]{2}{*}{ B20 } & & 898 & & & & \\
\hline & 5 & & 0 & -118 & -118 & 134 \\
\hline \multirow[t]{2}{*}{ B21 } & & 780 & & & & \\
\hline & 2 & & 93.0 & 74.0 & -19.0 & 105 \\
\hline \multirow[t]{2}{*}{ B22 } & & 854 & & & & \\
\hline & 7 & & 0 & 38.0 & 38.0 & 140 \\
\hline \multirow[t]{2}{*}{ B23 } & & 892 & & & & \\
\hline & 10 & & 0 & 28.0 & 28.0 & 145 \\
\hline \multirow[t]{2}{*}{ B24 } & & 920 & & & & \\
\hline & 7 & & 0 & 75.0 & 75.0 & 153 \\
\hline \multirow[t]{2}{*}{ B25 } & & 995 & & & & \\
\hline & 8 & & 0 & -152 & -152 & 164 \\
\hline \multirow[t]{2}{*}{ B26 } & & 843 & & & & \\
\hline & 15 & & 1.80 & 79.0 & 77.2 & 158 \\
\hline \multirow[t]{2}{*}{ B27 } & & 922 & & & & \\
\hline & 3 & & 0 & 19.0 & 19.0 & 149 \\
\hline \multirow[t]{2}{*}{ B28 } & & 941 & & & & \\
\hline & 14 & & 12.4 & 37.0 & 24.6 & 154 \\
\hline \multirow[t]{2}{*}{ B29 } & & 978 & & & & \\
\hline & 15 & & 20.8 & -15.0 & -35.8 & 155 \\
\hline \multirow[t]{2}{*}{ B30 } & & 963 & & & & \\
\hline & 7 & & 0 & 167 & 167 & 167 \\
\hline B31 & & 1,130 & & & & \\
\hline & 15 & & 0 & -40.0 & -40.0 & 178 \\
\hline B32 & & 1,090 & & & & \\
\hline & 8 & & 0 & -80.0 & -80.0 & 168 \\
\hline B33 & & 1,010 & & & & \\
\hline & 14 & & 3.48 & -136 & -139 & 151 \\
\hline B34 & & 874 & & & & \\
\hline & 6 & & 0 & 56.0 & 56.0 & 144 \\
\hline B35 & & 930 & & & & \\
\hline & 5 & & 0 & -59.0 & -59.0 & 144 \\
\hline
\end{tabular}


Table 8. Streamflow gains and losses computed for 27 reaches on the Brazos River, McLennan County to Fort Bend County, Texas, August 10-18, 2006.

[Bold font indicates gain or loss that is greater than potential measurement error for that particular reach. mi, miles; $\mathrm{ft}^{3} / \mathrm{s}$, cubic foot per second; --, not applicable]

\begin{tabular}{|c|c|c|c|c|c|c|}
\hline $\begin{array}{l}\text { Site } \\
\text { identi- } \\
\text { fier } \\
\text { (fig. 2) }\end{array}$ & $\begin{array}{c}\text { Distance between } \\
\text { downstream and } \\
\text { upstream sites } \\
\text { (mi) }\end{array}$ & $\begin{array}{c}\text { Streamflow, } \\
\text { instan- } \\
\text { taneous } \\
\left(\mathrm{ft}^{3} / \mathrm{s}\right)\end{array}$ & $\begin{array}{c}\text { Measured } \\
\text { inflow between } \\
\text { downstream and } \\
\text { upstream sites } \\
\left(\mathrm{ft}^{3} / \mathrm{s}\right) \\
\end{array}$ & $\begin{array}{c}\text { Difference in } \\
\text { streamflow between } \\
\text { downstream and } \\
\text { upstream sites } \\
\left(\mathrm{ft}^{3} / \mathrm{s}\right) \\
\end{array}$ & $\begin{array}{c}\text { Gain or loss in } \\
\text { streamflow between } \\
\text { downstream and } \\
\text { upstream sites } \\
\left(\mathrm{ft}^{3} / \mathrm{s}\right) \\
\end{array}$ & $\begin{array}{c}\text { Potential measurement } \\
\text { error between } \\
\text { downstream and } \\
\text { upstream sites } \\
\left(\mathrm{ft}^{3} / \mathrm{s}\right) \\
\end{array}$ \\
\hline \multirow[t]{2}{*}{ B1 } & -- & 370 & -- & -- & -- & -- \\
\hline & 26 & & 0.52 & -50.0 & -50.5 & 34.5 \\
\hline \multirow[t]{2}{*}{ B3 } & & 320 & & & & \\
\hline & 29 & & 1.00 & 76.0 & 75.0 & 35.8 \\
\hline \multirow[t]{2}{*}{ B6 } & & 396 & & & & \\
\hline & 17 & & 0 & 194 & 194 & 49.3 \\
\hline \multirow[t]{2}{*}{ B9 } & & 590 & & & & \\
\hline & 15 & & 0 & 39.0 & 39.0 & 61.0 \\
\hline \multirow[t]{2}{*}{ B12 } & & 629 & & & & \\
\hline & 5 & & 61.1 & -3.00 & -64.1 & 81.3 \\
\hline \multirow[t]{2}{*}{ B13 } & & 623 & & & & \\
\hline & 23 & & 0 & 134 & 134 & 87.7 \\
\hline \multirow[t]{2}{*}{ B14 } & & 757 & & & & \\
\hline & 17 & & 8.03 & -80.0 & -88.0 & 92.0 \\
\hline \multirow[t]{2}{*}{ B15 } & & 677 & & & & \\
\hline & 11 & & 0 & 73.0 & 73.0 & 71.3 \\
\hline \multirow[t]{2}{*}{ B16 } & & 750 & & & & \\
\hline & 15 & & 0 & 79.0 & 79.0 & 104 \\
\hline \multirow[t]{2}{*}{ B17 } & & 829 & & & & \\
\hline & 2 & & 6.59 & -18.0 & -24.6 & 131 \\
\hline \multirow[t]{2}{*}{ B18 } & & 811 & & & & \\
\hline & 2 & & 0 & 14.0 & 14.0 & 131 \\
\hline \multirow[t]{2}{*}{ B19 } & & 825 & & & & \\
\hline & 6 & & 0 & 138 & 138 & 143 \\
\hline \multirow[t]{2}{*}{ B20 } & & 963 & & & & \\
\hline & 5 & & 0 & -237 & -237 & 135 \\
\hline \multirow[t]{2}{*}{ B21 } & & 726 & & & & \\
\hline & 2 & & 0 & -24.0 & -24.0 & 114 \\
\hline \multirow[t]{2}{*}{ B22 } & & 702 & & & & \\
\hline & 7 & & 0 & 97.0 & 97.0 & 120 \\
\hline \multirow[t]{2}{*}{ B23 } & & 799 & & & & \\
\hline & 10 & & 0 & 26.0 & 26.0 & 130 \\
\hline \multirow[t]{2}{*}{ B24 } & & 825 & & & & \\
\hline & 7 & & 0 & -7.00 & -7.00 & 131 \\
\hline \multirow[t]{2}{*}{ B25 } & & 818 & & & & \\
\hline & 8 & & 0 & -11.0 & -11.0 & 130 \\
\hline \multirow[t]{2}{*}{ B26 } & & 807 & & & & \\
\hline & 15 & & 0 & 66.0 & 66.0 & 134 \\
\hline
\end{tabular}


Table 8. Streamflow gains and losses computed for 27 reaches on the Brazos River, McLennan County to Fort Bend County, Texas, August 10-18, 2006-Continued.

\begin{tabular}{|c|c|c|c|c|c|c|}
\hline $\begin{array}{l}\text { Site } \\
\text { identi- } \\
\text { fier } \\
\text { (fig. 2) }\end{array}$ & $\begin{array}{l}\text { Distance between } \\
\text { downstream and } \\
\text { upstream sites } \\
\text { (mi) }\end{array}$ & $\begin{array}{l}\text { Streamflow, } \\
\text { instan- } \\
\text { taneous } \\
\left(\mathrm{ft}^{3} / \mathbf{s}\right)\end{array}$ & $\begin{array}{c}\text { Measured } \\
\text { inflow between } \\
\text { downstream and } \\
\text { upstream sites } \\
\left(\mathrm{ft}^{3} / \mathrm{s}\right) \\
\end{array}$ & $\begin{array}{c}\text { Difference in } \\
\text { streamflow between } \\
\text { downstream and } \\
\text { upstream sites } \\
\left(\mathrm{ft}^{3} / \mathrm{s}\right) \\
\end{array}$ & $\begin{array}{c}\text { Gain or loss in } \\
\text { streamflow between } \\
\text { downstream and } \\
\text { upstream sites } \\
\left(\mathrm{ft}^{3} / \mathrm{s}\right) \\
\end{array}$ & $\begin{array}{c}\text { Potential measurement } \\
\text { error between } \\
\text { downstream and } \\
\text { upstream sites } \\
\left(\mathrm{ft}^{\mathrm{s}} / \mathrm{s}\right) \\
\end{array}$ \\
\hline \multirow[t]{2}{*}{ B27 } & & 873 & & & & \\
\hline & 3 & & 0 & -12.0 & -12.0 & 139 \\
\hline \multirow[t]{2}{*}{ B28 } & & 861 & & & & \\
\hline & 14 & & 3.40 & 40.0 & 36.6 & 141 \\
\hline \multirow[t]{2}{*}{ B29 } & & 901 & & & & \\
\hline & 15 & & 3.84 & -18.0 & -21.8 & 143 \\
\hline \multirow[t]{2}{*}{ B30 } & & 883 & & & & \\
\hline & 7 & & 0 & 1.00 & 1.00 & 141 \\
\hline \multirow[t]{2}{*}{ B31 } & & 884 & & & & \\
\hline & 15 & & 0 & -70.0 & -70.0 & 136 \\
\hline \multirow[t]{2}{*}{ B32 } & & 814 & & & & \\
\hline & 8 & & 0 & 56.0 & 56.0 & 135 \\
\hline \multirow[t]{2}{*}{ B33 } & & 870 & & & & \\
\hline & 20 & & -106 & -71.0 & -35.0 & 134 \\
\hline \multirow[t]{2}{*}{ B35 } & & 799 & & & & \\
\hline & 5 & & 0 & -103 & -103 & 120 \\
\hline B36 & & 696 & & & & \\
\hline
\end{tabular}

\section{Summary}

During 2006-07, the U.S. Geological Survey (USGS), in cooperation with the Texas Water Development Board (TWDB), did a study to quantify base flow and streamflow gains and losses in the Brazos River from McLennan County to Fort Bend County, Texas. This report documents the results of base-flow analysis (hydrograph separation) of historical (1966-2005) streamflow and two synoptic streamflow gain and loss surveys of the Brazos River from McLennan County to Fort Bend County, Texas, in March and August 2006.

The study area encompasses a 310-mile reach of the Brazos River from Waco in McLennan County to Richmond in Fort Bend County and includes selected inflow tributaries along the reach. The Brazos River is hydraulically connected to the Brazos River alluvium aquifer. Underlying the Brazos River alluvium aquifer in laterally adjacent layers generally parallel to the coast are two major aquifers, Carrizo-Wilcox and Gulf Coast, and three minor aquifers, Queen City, Sparta, and Yegua-Jackson. Upstream from the outcrop of the CarrizoWilcox aquifer, the Brazos River alluvium aquifer is underlain by rocks not characterized as part of a major or minor aquifer. The underlying aquifers are hydraulically connected to one another and to the Brazos River alluvium aquifer, as indicated by 1980 potentiometric-surface maps of the Carrizo-Wilcox, Queen City, and Sparta aquifers that show U-shaped potentiometric contours, closed upstream, across the Brazos River.

Hydrograph separation was done using the USGS computer program Hydrograph Separation and Analysis (HYSEP). The data used in HYSEP were historical (water years 19662005) streamflow from 10 USGS gaging stations, three on the Brazos River and seven on selected tributaries to the Brazos River. Streamflow data for computation of gains and losses were collected during March 6-10, 2006, from 36 sites on the Brazos River and 19 sites on 19 tributaries to the Brazos River; and during August 10-18, 2006, from 28 sites on the Brazos River and 16 sites on tributaries. HYSEP provides the base-flow index (BFI), which is the ratio of the annual mean base flow to the annual mean streamflow. The BFI was used as a means to graphically compare streamflows (hydrographs) from the three Brazos River stations. The graphical comparisons indicate whether the reach between two stations is gaining or losing flow.

Streamflow gains and losses for reaches defined by adjacent pairs of Brazos River measurement sites were computed as streamflow at the downstream site minus streamflow at the upstream site minus inflows from tributaries (plus a diversion for one reach). For this report, a stream reach is classified as verifiably gaining or losing only when the difference between 
streamflows at the upstream and downstream measuring sites that define the reach is greater than the potential error associated with the flow measurements.

Hydrograph separation and graphical evidence from BFI values indicate an appreciable increase in base flow as a percentage of streamflow in the reach of the Brazos River that crosses the outcrops of the Carrizo-Wilcox, Queen City, Sparta, and Yegua-Jackson aquifers compared to that in the adjacent upstream reach (on average from about 43 percent to about 60 percent). This result is consistent with indications of a gaining reach on 1980 potentiometric-surface maps of the Carrizo-Wilcox and Queen City-Sparta aquifers. Hydrograph separation and graphical evidence from BFI values also indicate no increase in base flow as a percentage of streamflow in the reach of the Brazos River crossing the Gulf Coast aquifer compared to that in the adjacent upstream reach.

Streamflow gains and losses computed for March 2006 for 35 reaches defined by pairs of sites on the Brazos River indicated that five reaches were verifiably gaining streamflow and none were verifiably losing streamflow. Four of the five gaining reaches are in the outcrop areas of the Carrizo-Wilcox and Yegua-Jackson aquifers. The reach gaining the most flow (258 cubic feet per second) is in the Yegua-Jackson aquifer outcrop. For the August survey, four stream reaches were verifiably gaining streamflow and two were losing streamflow. Although the August survey reaches are not everywhere comparable to the March survey reaches, the gaining reaches of the August survey generally match those of the March survey-occurring mostly in the outcrops of the aquifers. The reach gaining the most flow (194 cubic feet per second) begins upstream from the outcrop of the Carrizo-Wilcox aquifer and ends in the outcrop of that aquifer. The reach gaining the second-most flow (134 cubic feet per second) is in the outcrops of the Queen City and Sparta aquifers.

The results of the synoptic gain and loss surveys are consistent with the results of the base-flow analysis (hydrograph separation) of historical streamflow. Appreciable increases in streamflow, apparently the result of increases in base flow, occur in the reach of the Brazos River that crosses the outcrops of the Carrizo-Wilcox, Queen City, Sparta, and Yegua-Jackson aquifers. The gain and loss surveys also yielded no evidence for appreciable streamflow gains or losses in the outcrop of the Gulf Coast aquifer, except for one relatively large, unexplained loss (237 cubic feet per second) in the August survey.

\section{References}

Ashworth, J.B., and Hopkins, Janie, 1995, Major and minor aquifers of Texas: Texas Water Development Board Report $345,69 \mathrm{p}$.

Cronin, J.G., Follett, C.R., Shafer, G.H., and Rettman, P.L., 1963, Reconnaissance investigation of the ground-water resources of the Brazos River Basin, Texas: Texas Water Commission Bulletin 6310, 152 p.

Garza, Sergio, Jones, B.D., and Baker, E.T., Jr., 1987, Approximate potentiometric surfaces for the aquifers of the Texas Coastal Uplands system, 1980: U.S. Geological Survey Hydrologic Investigations Atlas, HA-704, 1 sheet, scale $1: 1,500,000$.

Helsel, D.R., and Hirsch, R.M., 1992, Statistical methods in water resources-Studies in environmental science 49: Amsterdam, Elsevier, 522 p.

National Weather Service, 2007, College Station extremes, normals and annual summaries: National Weather Service Forecast Office, Houston/Galveston, Texas, accessed March 29, 2007, at http://www.srh.noaa.gov/hgx/climate/cll/ normals/cll_summary.htm

Oberg, K.A., Morlock, S.E., and Caldwell, W.S., 2005, Quality-assurance plan for discharge measurements using acoustic Doppler current profilers: U.S. Geological Survey Scientific Investigations Report 2005-5183, 35 p.

Rantz, S.E., and others, 1982, Measurement and computation of streamflow-Volume 1. Measurement of stage and discharge: U.S. Geological Survey Water-Supply Paper 2175, chap. 5, p. 79-183.

Ryder, P.D., and Ardis, A.F., 2002, Hydrology of the Texas Gulf Coast aquifer systems, regional aquifer-system analysis-Gulf Coastal Plain: U.S. Geological Survey Professional Paper 1416-E, 77 p.

Sauer, V.B., and Meyer, R.W., 1992, Determination of error in individual discharge measurements: U.S. Geological Survey Open-File Report 92-144, 21 p.

Shah, S.D., Houston, N.A., and Braun, C.L., 2007, Hydrogeologic characterization of the Brazos River alluvium aquifer, Bosque County to Fort Bend County, Texas: U.S. Geological Survey Scientific Investigations Map 2989, 5 sheets.

Slade, R.M., Jr., Bentley, J.T., and Michaud, Dana, 2002, Results of streamflow gain-loss studies in Texas, with emphasis on gains from and losses to major and minor aquifers: U.S. Geological Survey Open-File Report 02-068, $131 \mathrm{p}$.

Sloto, R.A., and Crouse, M.Y., 1996, HYSEP_A computer program for streamflow hydrograph separation and analysis: U.S. Geological Survey Water-Resources Investigations Report 96-4040, 46 p.

Sontek/YSI, Inc., 2007, Argonaut-ADV and Flowtracker principles of operation, accessed on January 20, 2007, at http://www.sontek.com/princop/aadv/aadvpo.htm 
Texas Board of Water Engineers, 1960, Channel gain and loss investigations, Texas streams, 1918-1958: Texas Board of Water Engineers Bulletin 5807D, 270 p.

Texas Water Development Board, 2007, TWDB Mapping Website-Major aquifers, minor aquifers: accessed September 6, 2007, at http://www.twdb.state.tx.us/ mapping/.

U.S. Geological Survey, 2006, National Water Information System (NWIS Web) data available on the Word Wide Web: accessed March 2006 at http://waterdata.usgs.gov/tx/nwis/ nwis

U.S. Geological Survey, 2007, Water-resources data for the United States, water year 2006: U.S. Geological Survey
Water-Data Report WDR-US-2006, accessed March 1, 2007, at http://web10capp.er.usgs.gov/adr06_lookup/ search.jsp

Wahl, K.L., and Wahl, T.L, 1995, Determining the flow of Comal Springs at New Braunfels, Texas, in Texas Water '95, San Antonio, Texas, August 16-17, 1995, Proceedings: American Society of Civil Engineers, Texas Section, p. 77-86.

Wilde, F.D., and Radtke, D.B., 1998, National field manual for the collection of water-quality data-Field measurements: U.S. Geological Survey Techniques of Water-Resources Investigations, book 9, chap. A6 [variously paged].

Publishing support provided by:

Lafayette Publishing Service Center

Information regarding water resources in Texas is available at

http: //tx.usgs.gov/ 